\title{
Derivation of the propagation equations for higher order aberrations of local wavefronts
}

\author{
Gregor Esser, ${ }^{1, *}$ Wolfgang Becken, ${ }^{1}$ Werner Müller, ${ }^{1}$ Peter Baumbach, ${ }^{2}$ \\ Josep Arasa, ${ }^{3}$ and Dietmar Uttenweiler ${ }^{1}$ \\ ${ }^{1}$ Research and Development Ophthalmic Lenses, Rodenstock GmbH, Isartalstrasse 43, 80469 Munich, Germany \\ ${ }^{2}$ Aalen University, Beethovenstrasse 1, 73430 Aalen, Germany \\ ${ }^{3}$ Universitat Politècnica de Catalunya, Rambla Sant Nebridi 10, Terrassa 08222, Spain \\ *Corresponding author: gregor.esser@rodenstock.com
}

Received June 17, 2011; revised September 23, 2011; accepted September 23, 2011;

posted September 26, 2011 (Doc. ID 149379); published November 10, 2011

\begin{abstract}
From the literature the analytical calculation of local power and astigmatism of a wavefront after refraction and propagation is well known; it is, e.g., performed by the Coddington equation for refraction and the classical vertex correction formula for propagation. Recently the authors succeeded in extending the Coddington equation to higher order aberrations (HOA). However, equivalent analytical propagation equations for HOA do not exist. Since HOA play an increasingly important role in many fields of optics, e.g., ophthalmic optics, it is the purpose of this study to extend the propagation equations of power and astigmatism to the case of HOA (e.g., coma and spherical aberration). This is achieved by local power series expansions. In summary, with the results presented here, it is now possible to calculate analytically the aberrations of a propagated wavefront directly from the aberrations of the original wavefront containing both low-order and high-order aberrations. @ 2011 Optical Society of America OCIS codes: $\quad 000.3860,080.1005,080.1753,080.2720,080.7343,330.4460$.
\end{abstract}

\section{INTRODUCTION}

Aberrations play a decisive role in optics. In this work, we deal with them in the framework of geometrical optics in which the wavelength is neglected $(\lambda \rightarrow 0)$ with respect to diffraction effects [1,ㄹ]. Also in this case, the notions of both rays and wavefronts do still exist. A wavefront, in general defined as a surface of constant phase, is in this limit a surface of constant optical path length. A ray is a virtual infinitesimally small bundle of light, the direction of which is defined by the normal of the wavefront.

The analytical calculation of local power and astigmatism of a wavefront after refraction, and also propagation, is performed by the Coddington equation and the vertex correction formula. Recently the authors extended the Coddington equation to higher order aberrations (HOA) [3]. Therefore, with the equations in [3] it is possible to calculate analytically the HOA of an outgoing wavefront directly from the aberrations of the incoming wavefront and the refractive surface after refraction.

For calculating the wavefront aberrations of an entire optical system, it is necessary to propagate the wavefront from the intersection point of the chief ray at the first surface along the chief ray to the intersection point at the next surface, and so on. In the special case of a spectacle lens, this means the propagation from the front to the rear surface and further to the vertex point sphere or the entrance pupil of the eye. For second-order aberrations (power and astigmatism) the propagation of a wavefront is known and described by the analytical transfer equation, which can be described either in matrix form [4-8] or by power vectors [9]. Hitherto, for determining $\mathrm{HOA}$, the propagation of the wavefront was calculated by ray tracing $[\underline{1}, \underline{10}-13]$, which is an iterative numerical method.
The importance of wavefront driven correction of ocular aberrations, which are often measured by an aberrometer, has increased rapidly in recent years. The wavefront data are determined at some device-specific plane and by the diameter of the evaluated ray bundle. Depending on the desired application, it is usually necessary to transform these raw data to some other plane, e.g., the entrance pupil of the eye, the cornea (as is relevant for LASIK or contact lenses), or the vertex plane of a spectacle lens. The ray bundle's diameter, in turn, is determined by the pupil size of the eye.

While there exist various publications dealing with analytical scaling transformations to a different pupil size [14-21], rotating the pupil [14,20-22], displacing the pupil $[\underline{14}, \underline{15}, 20-22]$, or deforming the pupil [21], only a few publications can be found that attempt to treat the wavefront propagation in an analytical way. In [14,23], an analytical method is described to calculate the propagation of a wavefront, but the method is still restricted by some approximations. As is written there, further study is necessary to obtain a unified formulation for wavefronts containing both low-order and highorder aberrations. In this work we have developed such a unified analytical propagation method in homogenous material.

\section{METHODS AND THEORETICAL BACKGROUND}

\section{A. Definitions and Notation}

It turns out to be very practical to establish the treatment of propagation, including HOA, on the basis of wavefront sagittas in space and not directly with optical path difference (OPD)-based aberrations. A connection between those two pictures is provided in [3]]. In contrast to [3] , where three coordinate systems were appropriate for describing refraction, in the case of propagation where tilt is absent, it is practical to 
use one common global Cartesian coordinate system $(x, y, z)$ in order to describe the original wavefront and the propagated wavefront. The system is defined by the intersection point of the chief ray with the original wavefront and by the direction of the chief ray, which defines the $z$ axis. The orientation of the $x$ axis can be freely selected. The orientation of the $y$ axis is such that the system is right-handed (see Fig. 1).

In this publication the wavefront description, the relation between the coefficients and the derivatives, and also the connection between coefficients and local aberrations are defined in the same way as used in [3].

\section{B. Local Properties of Wavefronts}

Considering the infinitesimal area around the optical axis, or rather around the chief ray, leads to Gaussian optics (or paraxial optics) [1]. For the aberrations of second order, the propagation of a spherical wavefront with the power $S_{o}$ (see Fig. 1) is described by the propagation or transfer equation [4-9]]:

$$
S_{p}=\frac{1}{1-\frac{d}{n} S_{o}} S_{o}
$$

where

$S_{o}=n / s_{o}$ is the vergence of the original wavefront.

$S_{p}=n / s_{p}$ is the vergence of the propagated wavefront.

$s_{o}$ is the vertex distance of the original wavefront (distance along the chief ray from the wavefront to the image point), which is equivalent to the radius of curvature of the original wavefront.

$s_{p}$ is the vertex distance of the propagated wavefront (distance along the chief ray from the wavefront to the image point), which is equivalent to the radius of curvature of the propagated wavefront.

$n$ is the refractive index.

$d$ is the propagation distance.

In the literature, the notion of vergence is usually extended to three-dimensional (3D) space for describing the spherocylindrical power of a wavefront in terms of $2 \times 2$ vergence matrices $[3,24,25]$ of the shape

$$
n\left(\begin{array}{ll}
w_{o}^{(2,0)} & w_{o}^{(1,1)} \\
w_{o}^{(1,1)} & w_{o}^{(2,0)}
\end{array}\right)
$$

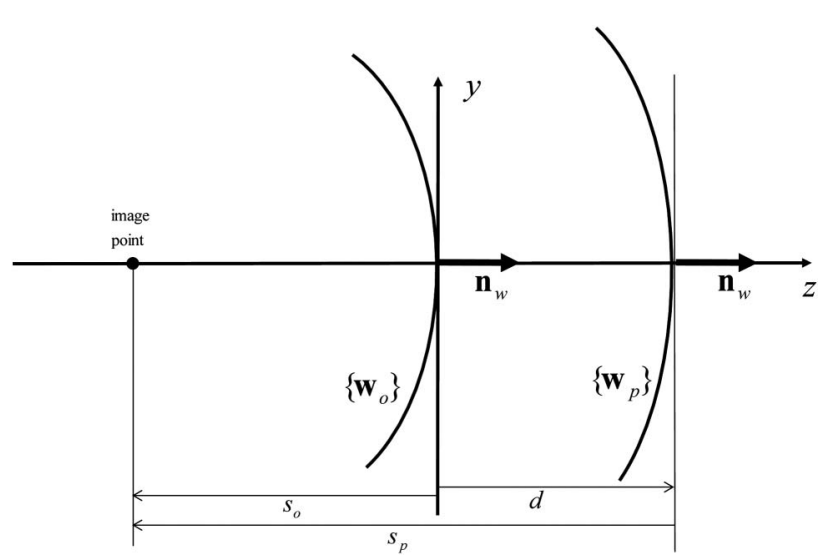

Fig. 1. Propagation of a spherical wavefront $\mathbf{w}_{o}$ with a vergence distance $s_{o}$ about the distance $d$ to the propagated wavefront $\mathbf{w}_{p}$ with a vergence distance $s_{p}$. for $w_{o}(x, y)$, and similarly for $w_{p}(x, y)$ :

$$
\begin{aligned}
& \mathbf{S}_{o}=\left(\begin{array}{ll}
S_{o, x x} & S_{o, x y} \\
S_{o, x y} & S_{o, y y}
\end{array}\right)=n\left(\begin{array}{ll}
w_{o}^{(2,0)} & w_{o}^{(1,1)} \\
w_{o}^{(1,1)} & w_{o}^{(0,2)}
\end{array}\right), \\
& \mathbf{S}_{p}=\left(\begin{array}{ll}
S_{p, x x} & S_{p, x y} \\
S_{p, x y} & S_{p, y y}
\end{array}\right)=n\left(\begin{array}{ll}
w_{p}^{(2,0)} & w_{p}^{(1,1)} \\
w_{p}^{(1,1)} & w_{p}^{(0,2)}
\end{array}\right) .
\end{aligned}
$$

The relation between the components of Eq. (2) and the ophthalmic terms sph, cyl, axis are well known [3]].

One well-established generalization of Eq. (1) relating the components of Eq. (2) to each other is the "generalized propagation equation." It describes the propagation of an astigmatic wavefront [4-8], written in compact form in terms of vergence matrices:

$$
\mathbf{S}_{p}=\frac{1}{1-\frac{d}{n} \mathbf{S}_{o}} \mathbf{S}_{o},
$$

where we have introduced the unit matrix

$$
\mathbf{1}=\left(\begin{array}{ll}
1 & 0 \\
0 & 1
\end{array}\right) .
$$

In addition to the description in terms of vergence matrices, an equivalent description is common in the $3 \mathrm{D}$ vector space of power vectors $[\underline{24}, \underline{26}]$. In [9] also, the "generalized propagation equation" in terms of power vectors is described.

Analogously to the definition of the power vectors for aberrations of order $k=2$, similar vectors $\mathbf{e}_{k}$ of dimension $k+1$ for aberrations of higher order $k>2$ have been defined [3].

\section{Mathematical Approach in the Two-Dimensional (2D) Case}

1. Description of Wavefronts in the 2D Case

The wavefronts themselves are each described by power series expansions. Any point on the original wavefront is given by the vector

$$
\mathbf{w}_{o}(y)=\left(\begin{array}{c}
y \\
w_{o}(y)
\end{array}\right),
$$

where in the $2 \mathrm{D}$ case $w_{o}(y)$ is the curve defined by

$$
w_{o}(y)=\sum_{k=0}^{\infty} \frac{a_{o, k}}{k !} y^{k}
$$

The normal vectors and their derivatives are described as in [3] and obey the same relations as therein in Eqs. (22)-(24) [Appendix A Eqs. (A1) and (A2)]. Since the normal vector of the original wavefront and the normal vector of the propagated wavefront are equal, the normal vector will be labeled generally with $\mathbf{n}_{w}$.

In application on the functions of interest, $\mathbf{n}_{w}(y)=$ $\mathbf{n}\left(w_{o}^{(1)}(y)\right)$; this means that $\mathbf{n}_{w}(0)=(0,1)^{T}$. Further, the first derivatives are given by 


$$
\left.\frac{\partial}{\partial y} \mathbf{n}_{w}(y)\right|_{y=0} \equiv \mathbf{n}_{w}^{(1)}(0)=\mathbf{n}^{(1)}(0) w_{o}^{(2)}(0)=\left(\begin{array}{c}
-1 \\
0
\end{array}\right) w_{o}^{(2)}(0),
$$

and similarly for the higher derivatives.

\section{Ansatz for Determining the Propagation Equations}

Once the local aberrations of the original wavefront are given, its corresponding coefficients $\alpha_{k}$ are directly determined, too, and equivalently the wavefront's derivatives. It is our aim to calculate the propagated wavefront, in the sense that its derivatives and thus its $a_{k}$ coefficients [see Eqs. (5) and (ㅁ)] are determined for all orders $2 \leq k \leq k_{0}$ for the order $k_{0}$ of interest, and to assign values to its corresponding local aberrations.

In contrast to this general procedure, which is the same as in [3], we now consider the following situation as a starting point for treating propagation. While the chief ray and the coordinate system are fixed, a neighboring ray scans the original wavefront $\left\{\mathbf{w}_{o}\right\}$ and hits it at an intercept $y_{o} \neq 0$, then propagates to the propagated wavefront $\left\{\mathbf{w}_{p}\right\}$, where the brackets $\{\cdot\}$ shall denote the entity of vectors described by Eq. (ㅁ). As shown in Fig. 2, and consistently with our notation, we denote as $y_{o}$ the projection of the neighboring ray's intersection with $\left\{\mathbf{w}_{o}\right\}$ onto the $y$ axis, and analogously, the projection of the intersection with $\left\{\mathbf{w}_{p}\right\}$ onto the $y$ axis is denoted as $y_{p}$.

The vector $\mathbf{w}_{o}=\mathbf{w}_{o}\left(y_{o}\right)$ [see Eq. (5)] points to the neighboring ray's intersection point with the original wavefront, and the propagated wavefront's OPD, referred to the original wavefront measured along the ray, is denoted by $\tau$. Correspondingly the vector from the original wavefront to the propagated wavefront is $\frac{\tau}{n} \mathbf{n}_{w}$. Hence, the vector to the point on the propagated wavefront itself, $\mathbf{w}_{p}$, must be equal to the vector sum $\mathbf{w}_{p}=\mathbf{w}_{o}+\frac{\tau}{n} \mathbf{n}_{w}$. This yields the fundamental equation

$$
\left(\begin{array}{c}
y_{o} \\
w_{o}\left(y_{o}\right)
\end{array}\right)+\frac{\tau}{n} \mathbf{n}_{w}=\left(\begin{array}{c}
y_{p} \\
w_{p}\left(y_{p}\right)
\end{array}\right)
$$

From Eq. (8), it is now possible to derive the desired relations order by order. Although only the propagated wavefront is of interest, in Eq. (8) additionally the quantities $y_{o}$ and $y_{p}$ are also unknown. However, those are not independent from each

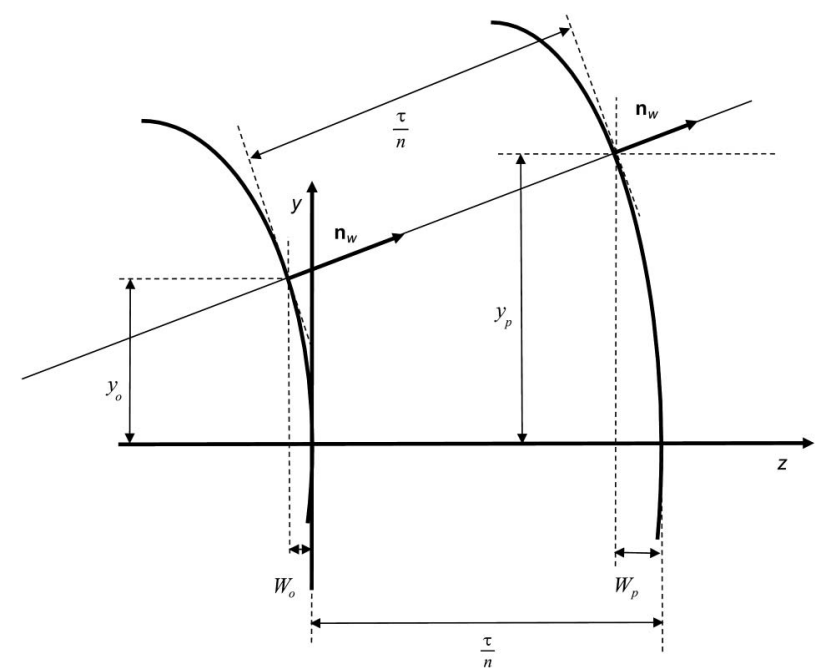

Fig. 2. Propagation of a wavefront $\mathbf{w}_{o}$ about the distance $d=\frac{\tau}{n}$ to the propagated wavefront $\mathbf{w}_{p}$. other; if any one of them is given, the other one can no longer be chosen independently. The coordinate $y_{p}$ is used as an independent variable, and $y_{0}$ is considered as a function of it.

Equation ( $\underline{8}$ ) represents a nonlinear system of two algebraic equations for the two unknown functions $w_{p}\left(y_{p}\right)$ and $y_{o}\left(y_{p}\right)$. Even if we are only interested in a solution for the function $w_{p}\left(y_{p}\right)$, we cannot obtain it without simultaneously solving the equations for both unknowns order by order. Introducing the vector of unknown functions as

$$
\mathbf{p}\left(y_{p}\right)=\left(\begin{array}{c}
y_{o}\left(y_{p}\right) \\
w_{p}\left(y_{p}\right)
\end{array}\right)
$$

and observing that the initial condition $\mathbf{p}(0)=(0, \tau / n)^{T}$ has to be fulfilled, it is now straightforward to compute all the derivatives of Eq. (8) up to some order, which yields relations between the curvatures, third derivatives, etc., of the original and propagated wavefront. Rewriting these relations in terms of series coefficients $a_{o, k}$ and solving them for the desired coefficients $a_{p, k}$ yields the desired result.

Rewriting Eq. (ㅁ) leads to

$$
\left(\begin{array}{l}
0 \\
0
\end{array}\right)=\left(\begin{array}{c}
y_{o}+\frac{\tau}{n} n_{w, y}-y_{p} \\
w_{o}\left(y_{o}\right)+\frac{\tau}{n} n_{w, z}-w_{p}\left(y_{p}\right)
\end{array}\right)
$$

Before solving Eq. (10), we distinguish if the independent variable $y_{p}$ enters into Eq. (10) explicitly, like in the first component of the vector $\left(y_{p}, w_{p}\left(y_{p}\right)\right)^{T}$, or implicitly via one of the components of Eq. (9). To this end, we follow the concept of [3] and define in this case the function $\left(\mathbb{R}^{2} \times \mathbb{R}\right) \mapsto \mathbb{R}^{2}$ : $\left(\mathbf{p}, y_{p}\right) \mapsto \mathbf{f}$ by

$$
\mathbf{f}\left(\mathbf{p}, y_{p}\right)=\left(\begin{array}{c}
y_{o}+\frac{\tau}{n} n_{w, y}\left(w_{o}^{(1)}\left(y_{o}\right)\right)-y_{p} \\
w_{o}\left(y_{o}\right)+\frac{\tau}{n} n_{w, z}\left(w_{o}^{(1)}\left(y_{o}\right)\right)-w_{p}
\end{array}\right),
$$

where $\left(p_{1}, p_{2}\right)=\left(y_{o}, w_{p}\right)$ are the components of p. Setting $\mathbf{p}=\mathbf{p}\left(y_{p}\right)$, Eq. (11) allows us to rewrite the fundamental system of Eq. (10) in a more compact way as

$$
\mathbf{f}\left(\mathbf{p}\left(y_{p}\right), y_{p}\right)=\mathbf{0}
$$

as can be verified explicitly by componentwise comparison with Eq. (10).

Solving Eq. (12) for the function $\mathbf{p}\left(y_{p}\right)$ is formally identical to solving Eq. (28) in [3]. The only difference is that now the name of the independent variable is $y_{p}$ instead of $\bar{y}_{S}$ in [3]. Taking the total derivative of Eq. (12) with respect to $y_{p}$ and applying the principles from the theory of implicit functions leads to the system of differential equations

$$
\sum_{j=1}^{2} \frac{\partial f_{i}}{\partial p_{j}} p_{j}^{(1)}\left(y_{p}\right)+\frac{\partial f_{i}}{\partial y_{p}}=0, \quad i=1,2,
$$

where the matrix with elements $A_{i j}:=\partial f_{i} / \partial p_{j}$ is the Jacobian matrix $\mathbf{A}$ of $\mathbf{f}$ with respect to its vector argument $\mathbf{p}$, evaluated for $\mathbf{p}=\mathbf{p}\left(y_{p}\right)$. The Jacobian A reads

$$
\mathbf{A}:=\left(\begin{array}{ll}
\frac{\partial f_{1}}{\partial y_{o}} & \frac{\partial f_{1}}{\partial w_{p}} \\
\frac{\partial f_{2}}{\partial y_{o}} & \frac{\partial f_{2}}{\partial w_{p}}
\end{array}\right)=\left(\begin{array}{cc}
1+\frac{\tau}{n} n_{w, y}^{(1)} w_{o}^{(2)} & 0 \\
w_{o}^{(1)}+\frac{\tau}{n} n_{w, z}^{(1)} w_{o}^{(2)} & -1
\end{array}\right) .
$$


In Eq. (14), the occurring expressions are understood as $w_{o}^{(1)} \equiv w_{o}^{(1)}\left(y_{o}\right), w_{o}^{(2)} \equiv w_{o}^{(2)}\left(y_{o}\right), n_{w, y} \equiv n_{w, y}\left(w_{o}^{(1)}\left(y_{o}\right)\right), n_{w, y}^{(1)} \equiv$ $n_{w, y}^{(1)}\left(w_{o}^{(1)}\left(y_{o}\right)\right)$, etc., and in addition, $y_{o}$ and $w_{p}$ are themselves functions of $y_{p}$.

The derivative vector $\partial f_{i} / \partial y_{p}$ in Eq. (13) shall be summarized as

$$
\mathbf{b}:=-\frac{\partial \mathbf{f}}{\partial y_{p}}=\left(\begin{array}{l}
1 \\
0
\end{array}\right)
$$

Similarly to [3] , we conclude that we can write $\mathbf{A}$ with argument $\mathbf{A}\left(\mathbf{p}\left(y_{p}\right)\right)$ only and $\mathbf{b}$ without argument at all because $\mathbf{b}$ is constant.

Equation (13) can then be written in the form

$$
\mathbf{A}\left(\mathbf{p}\left(y_{p}\right)\right) \mathbf{p}^{(1)}\left(y_{p}\right)=\mathbf{b}
$$

\section{Solving Techniques for the Fundamental Equation}

For solving Eq. (16) for $\mathbf{p}\left(y_{p}\right)$, we can apply the same exact steps as in Eqs. (33)-(36) in [3], with the only difference that here the independent variable is named $y_{p}$ instead of $\bar{y}_{S}$, and also that the initial condition here reads $\mathbf{p}(0)=(0, \tau / n)^{T}$ instead of $\mathbf{p}(0)=0$ as was the case in [3]. The equations as a function of the independent variable $y_{p}$ are shown in Appendix A [Eqs. (A3)-(A6)]. Hence, in this paper, we directly provide a formal solution for $\mathbf{p}^{(k)}(0)$, expressed in terms of its predecessors, by the equations

$$
\begin{aligned}
& \mathbf{p}^{(1)}(0)=\mathbf{A}^{-1} \mathbf{b}, \quad k=1, \\
& \mathbf{p}^{(k)}(0)=-\mathbf{A}^{-1} \sum_{j=1}^{k-1}\left(\begin{array}{c}
k-1 \\
j-1
\end{array}\right) \mathbf{A}^{(k-j)} \mathbf{p}^{(j)}(0), \quad k \geq 2,
\end{aligned}
$$

where $\mathbf{A}^{-1}=\mathbf{A}(\mathbf{p}(0))^{-1}=\mathbf{A}(\mathbf{0})^{-1}$.

\section{Solutions for the General Propagation Equations}

In the result for $\mathbf{p}^{(1)}(0)$, the first rows of Eq. (17) involve $\mathbf{A}(\mathbf{0})^{-1}$. For obtaining $\mathbf{A}(\mathbf{0})^{-1}$, we evaluate Eq. (14) for $\mathbf{p}=\mathbf{0}$ and apply Eq. (23) in [3] [Eq. (르) in Appendix $\underline{\mathrm{A}}$ ], yielding

$$
\mathbf{A}(\mathbf{0})=\left(\begin{array}{cc}
1-\frac{\tau}{n} w_{o}^{(2)} & 0 \\
0 & -1
\end{array}\right) \Rightarrow \mathbf{A}(\mathbf{0})^{-1}=\left(\begin{array}{cc}
\frac{1}{1-\frac{\tau}{n} w_{o}^{(2)}} & 0 \\
0 & -1
\end{array}\right) .
$$

The final result for $\mathbf{p}^{(1)}(0)$ is

$$
\mathbf{p}^{(1)}(0)=\mathbf{A}^{-1} \mathbf{b}=\left(\begin{array}{c}
\frac{1}{1-\frac{\tau}{n} w_{o}^{(2)}} \\
0
\end{array}\right)
$$

The first derivative of the $y_{0}$ coordinate, which is the first component $p_{1}$ of $\mathbf{p}$, is a dilation depending on the curvature of the original wavefront and the propagated optical path length $\tau$, such that $y_{o}^{(1)}(0)=\frac{1}{1-(\tau / n) w_{0}^{(2)}}$. The slope of the propagated wavefront vanishes, $w_{p}^{(1)}(0)=0$, as does the slope of the original wavefront due to $w_{o}^{(1)}(0)=0$.

For the orders $k \geq 2$, we apply Eq. (17). The derivatives $\mathbf{A}^{(1)}=\left.\frac{d}{d y_{p}} \mathbf{A}\left(\mathbf{p}\left(y_{p}\right)\right)\right|_{y_{p}=0}$, etc., are directly obtained by the total derivative of Eq. ( $\underline{14})$ with respect to $y_{p}$, evaluating for $y_{p}=0$ and again applying Eq. (A2). For the orders $k \geq 2$, only the results $w_{p}^{(k)}(0)$ for the propagated wavefront are of interest; therefore, we directly provide those results. The resulting second-order law is [omitting the argument "(0)"]

$$
w_{p}^{(2)}=\beta w_{o}^{(2)}
$$

with

$$
\beta=\frac{1}{1-\frac{\tau}{n} w_{o}^{(2)}}
$$

which is well known as the propagation equation and appears to be a special case of the results. The resulting higher-order laws can be written in a similar fashion:

$$
\begin{aligned}
& w_{p}^{(3)}=\beta^{3} w_{o}^{(3)}, \quad w_{p}^{(4)}=\beta^{4}\left(w_{o}^{(4)}+3 \frac{\tau}{n}\left(\beta w_{o}^{(3)^{2}}-w_{o}^{(2)^{4}}\right)\right), \\
& w_{p}^{(5)}=\beta^{5}\left(w_{o}^{(5)}+5 \beta \frac{\tau}{n} w_{o}^{(3)}\left(2 w_{o}^{(4)}+3 \beta \frac{\tau}{n} w_{o}^{(3)^{2}}-6 w_{o}^{(2)^{3}}\right)\right),
\end{aligned}
$$

Equation (22) can be generalized for $2<k \leq 6$ to

$$
w_{p}^{(k)}=\beta^{k}\left(w_{o}^{(k)}+R_{k}\right),
$$

where in $R_{k}$ the dependence of $w_{p}^{(k)}$ on all wavefront derivatives $w_{o}^{(j)}$ of lower order $(j<k)$ is summarized.

\section{Special Case}

Although application of Eq. (17) provides a solution for $w_{p}^{(k)}(0)$ up to arbitrary order $k$, it is very instructive to analyze the solutions more closely for one special case. We observe that the expressions in Eq. (23) for $R_{k}$ will vanish if we set $w_{o}^{(j)}=0$ for all lower orders $j<\bar{k}$ (for $k=3$ or $k=4$, respectively).

This leads to the assumption (for $k>2$ ) that the following statement is generally true: if only aberrations for one single given order $k$ are present, while for all lower orders $j<k$ we have $w_{o}^{(j)}=0$, then $\left(\frac{1}{1-(\tau / n) w_{o}^{(2)}}\right)=1$ and $R_{k}=0$, which means that for fixed order $k$ Eq. (23) will be valid for the vanishing remainder term and the aberration of the propagated wavefront will be equal to the aberration of the original wavefront independent of the propagation distance $d$.

To this purpose, we start from the recursion scheme in Eq. (17) and show that only the term containing $\mathbf{p}^{(1)}$ can contribute to the sum if all aberrations vanish for orders less than $k$. For doing so, it is necessary to exploit two basic properties of the derivatives $\mathbf{A}^{(m)}=\left.\frac{d^{m}}{d y_{p}^{m}} \mathbf{A}\left(\mathbf{p}\left(y_{p}\right)\right)\right|_{y_{p}=0}$ of the matrix $\mathbf{A}$ for the orders $1 \leq m \leq k-1$. As can be shown by elementwise differentiation of the matrix $\mathbf{A}$, the highest wavefront derivatives present in $\mathbf{A}^{(m)}\left(\mathbf{p}\left(y_{p}\right)\right)$ [see Eq. (14)] are proportional to $w_{o}^{(m+2)}$. Evaluating $\mathbf{A}^{(m)}\left(\mathbf{p}\left(y_{p}\right)\right)$ at the position $y_{p}=0$ shows that $\mathbf{A}^{(m)}$ cannot contain any higher wavefront derivatives than $w_{o}^{(m+2)}$. It follows that

i. The highest possible wavefront derivatives present in $\mathbf{A}^{(m)}$ are $w_{o}^{(m+2)}$.

ii. If all wavefront derivatives, even up to order $(m+2)$, vanish, then $\mathbf{A}^{(m)}$ itself will vanish. This is in contrast to $\mathbf{A}$ itself, which contains constants and therefore will be finite 
even if all wavefront derivatives vanish.

Analyzing the terms in Eq. (17), we notice that the occurring derivatives of the matrix $\mathbf{A}$ are $\mathbf{A}^{(k-1)}, \mathbf{A}^{(k-2)}, \ldots, \mathbf{A}^{(2)}, \mathbf{A}^{(1)}$ for $j=1,2, \ldots,(k-1)$, respectively. It follows from property (i) that the highest occurring wavefront derivatives in these terms are $(k+1), k,(k-1), \ldots, 3,2$, respectively. Now, if all wavefront derivatives up to order $(k-1)$ vanish, it will follow from property (ii) that all the matrix derivatives $\mathbf{A}^{(k-3)}, \ldots, \mathbf{A}^{(2)}, \mathbf{A}^{(1)}$ must vanish, leaving only $\mathbf{A}^{(k-1)}$ and $\mathbf{A}^{(k-2)}$. Therefore all terms in Eq. (17) vanish, excluding only the contribution for $j=1$ and $j=2$. We directly conclude that

$$
\begin{aligned}
& \mathbf{p}^{(2)}=-\mathbf{A}^{-1} \mathbf{A}^{(1)} \mathbf{A}^{-1} \mathbf{b}, \quad k=2, \\
& \mathbf{p}^{(k)}=-\mathbf{A}^{-1} \sum_{j=1}^{2}\left(\begin{array}{c}
k-1 \\
j-1
\end{array}\right) \mathbf{A}^{(k-j)} \mathbf{p}^{(j)}, \quad k \geq 3 .
\end{aligned}
$$

This leads directly to

$$
\begin{aligned}
& \mathbf{p}^{(2)}=-\mathbf{A}^{-1} \mathbf{A}^{(1)} \mathbf{A}^{-1} \mathbf{b}, \quad k=2, \\
& \mathbf{p}^{(k)}=\mathbf{A}^{-1}\left((k-1) \mathbf{A}^{(k-2)} \mathbf{A}^{-1} \mathbf{A}^{(1)}-\mathbf{A}^{(k-1)}\right) \mathbf{A}^{-1} \mathbf{b}, \quad k \geq 3 .
\end{aligned}
$$

In the term

$$
\mathbf{A}^{(1)}=\left(\begin{array}{cc}
\frac{-\frac{\tau}{n}}{1-\frac{\tau}{n} w_{o}^{(2)}} w_{o}^{(3)} & 0 \\
w_{o}^{(2)} & 0
\end{array}\right)
$$

only wavefront derivatives $w_{o}^{(3)}$ and $w_{o}^{(2)}$ occur. Therefore $\mathbf{A}^{(1)}=\mathbf{0}$ for $k>3$ because $w_{o}^{(3)}$ and $w_{o}^{(2)}$ vanish. Equation (25) can then be written in the form

$\mathbf{p}^{(3)}=\mathbf{A}^{-1}\left(2 \mathbf{A}^{(1)} \mathbf{A}^{-1} \mathbf{A}^{(1)}-\mathbf{A}^{(2)}\right) \mathbf{A}^{-1} \mathbf{b}, \quad k=3$,

$\mathbf{p}^{(k)}=-\mathbf{A}^{-1} \mathbf{A}^{(k-1)} \mathbf{A}^{-1} \mathbf{b}, \quad$ otherwise.

To evaluate $\mathbf{p}^{(3)}$ in Eq. (27), the second derivative of $\mathbf{A}$ has to be calculated. $\mathbf{A}^{(2)}$ reads, if all derivatives of the wavefront vanish for orders less than or equal to $m=2$,

$$
\mathbf{A}^{(2)}=\left(\begin{array}{cc}
-\frac{\tau}{n}\left(\frac{\tau}{n} w_{o}^{(3)}+w_{o}^{(4)}\right) & 0 \\
w_{o}^{(3)} & 0
\end{array}\right) .
$$

For evaluating $\mathbf{A}^{(k-1)}$ for $k-1>2$, we set $k-1=: m$, and it is straightforward to show by induction that if all aberrations vanish for orders less than or equal to $m$, then

$$
\mathbf{A}^{(m)}=\left(\begin{array}{cc}
-\frac{\tau}{n} w_{o}^{(m+2)} & 0 \\
w_{o}^{(m+1)} & 0
\end{array}\right)
$$

where $y_{o}^{(1)}$ has been substituted by their solution $\frac{1}{1-(\tau / n) w_{o}^{(2)}}$ wherever it occurs [see Eq. (19)]. Inserting $\mathbf{A}^{(m)}(0)$ for $m=$ $k-1$ and $\mathbf{A}(0)^{-1}$ from Eq. (18) into Eq. (27) directly yields that

$$
w_{p}^{(k)}=w_{o}^{(k)}
$$

for all orders $k>2$.

\section{Mathematical Approach in the 3D Case}

1. Wavefronts and Normal Vectors

Although it is more lengthy to demonstrate than the 2D case, conceptually the $3 \mathrm{D}$ case can be treated analogously to the $2 \mathrm{D}$ case and analogously to Eqs. (49)-(59) in [3]. Therefore, we will only report the most important differences. Analogously to Eq. (5), the original wavefront is now represented by the $3 \mathrm{D}$ vector

$$
\mathbf{w}_{o}(x, y)=\left(\begin{array}{c}
x \\
y \\
w_{o}(x, y)
\end{array}\right)
$$

where $w_{o}(x, y)$ and the relation between the coefficients and the derivatives are defined as described in [3]. The connection between coefficients and local aberrations is given by multiplying the coefficient with the refractive index.

For treating the normal vectors, we use the same function,

$$
\mathbf{n}(u, v):=\frac{1}{\sqrt{1+u^{2}+v^{2}}}\left(\begin{array}{c}
-u \\
-v \\
1
\end{array}\right),
$$

as in [3] and make use of the fact that the normal vector $\mathbf{n}_{w}(x, y)$ to a surface $\mathbf{w}(x, y):=(x, y, w(x, y))^{T}$ is given by $\mathbf{n}(\nabla w)$. In the intersection point we now have $\mathbf{n}_{w}(0,0)=(0,0,1)^{T}$, and the derivatives corresponding to Eq. (A2) can be directly obtained from Eq. (32).

\section{Ansatz for Determining the Propagation Equations}

The starting point for establishing the relations between the original and the propagated wavefront is now given by equations analogous to Eq. (8), with the only difference that the $x$ and $y$ components are simultaneously present.

The vector of unknown functions is now given by

$$
\mathbf{p}\left(x_{p}, y_{p}\right)=\left(\begin{array}{c}
x_{o}\left(x_{p}, y_{p}\right) \\
y_{o}\left(x_{p}, y_{p}\right) \\
w_{p}\left(x_{p}, y_{p}\right)
\end{array}\right)
$$

and the 3D analogue to Eq. (8) now leads to

$$
\mathbf{f}\left(\mathbf{p}\left(x_{p}, y_{p}\right), x_{p}, y_{p}\right)=\mathbf{0},
$$

where $\mathbf{f}$ is the $3 \mathrm{D}$ analogue to Eq. (11).

Since Eq. (34) is formally identical to Eq. (52) in [3], the solving procedure from [3] can be directly applied. In particular, we have to deal with two first-order equations:

$$
\begin{aligned}
& \mathbf{A}\left(\mathbf{p}\left(x_{p}, y_{p}\right)\right) \mathbf{p}^{(1,0)}\left(x_{p}, y_{p}\right)=\mathbf{b}_{x}, \\
& \mathbf{A}\left(\mathbf{p}\left(x_{p}, y_{p}\right)\right) \mathbf{p}^{(0,1)}\left(x_{p}, y_{p}\right)=\mathbf{b}_{y},
\end{aligned}
$$

which correspond exactly to Eq. (53) in [3]. Of course, the explicit expressions regarding how $\mathbf{f}$ and $\mathbf{p}$ depend on their arguments now lead to different expressions for the column vectors of the inhomogeneities,

$$
\mathbf{b}_{x}=-\frac{\partial \mathbf{f}}{\partial x_{p}}=\left(\begin{array}{lll}
1 & 0 & 0
\end{array}\right)^{T}, \quad \mathbf{b}_{y}=-\frac{\partial \mathbf{f}}{\partial y_{p}}=\left(\begin{array}{lll}
0 & 1 & 0
\end{array}\right)^{T},
$$

and for the Jacobian matrix $\mathbf{A}\left(\mathbf{p}\left(x_{p}, y_{p}\right)\right)$ with elements $A_{i j}:=\partial f_{i} / \partial p_{j}$, which is now given by 


$$
\mathbf{A}\left(\mathbf{p}\left(x_{p}, y_{p}\right)\right)=\left(\begin{array}{ccc}
1+\frac{\tau}{n}\left(n_{w, x}^{(0,1)} w_{o}^{(1,1)}+n_{w, x}^{(1,0)} w_{o}^{(2,0)}\right) & \frac{\tau}{n}\left(n_{w, x}^{(0,1)} w_{o}^{(0,2)}+n_{w, x}^{(1,0)} w_{o}^{(1,1)}\right) & 0 \\
\frac{\tau}{n}\left(n_{w, y}^{(0,1)} w_{o}^{(1,1)}+n_{w, y}^{(1,0)} w_{o}^{(2,0)}\right) & 1+\frac{\tau}{n}\left(n_{w, y}^{(0,1)} w_{o}^{(0,2)}+n_{w, y}^{(1,0)} w_{o}^{(1,1)}\right) & 0 \\
w_{o}^{(1,0)}+\frac{\tau}{n}\left(n_{w, z}^{(0,1)} w_{o}^{(1,1)}+n_{w, z}^{(1,0)} w_{o}^{(2,0)}\right) & w_{o}^{(0,1)}+\frac{\tau}{n}\left(n_{w, z}^{(0,1)} w_{o}^{(0,2)}+n_{w, z}^{(1,0)} w_{o}^{(1,1)}\right) & -1
\end{array}\right)
$$

\section{Solutions for the General Propagation Equations}

The formal analogy of Eq. (35) to Eq. (53) in [3] can be exploited by making use of the solving techniques developed in [3]. Equivalently, either Eq. (58) or Eqs. (63a)-(63d) from [3] [Eqs. (A7) and (A8) in Appendix A] can be directly applied. The only difference from [3] is now that again the explicit expressions for the Jacobian and its inverse, which have to be inserted into Eqs. (58) or Eqs. (63a)-(63d) from [3], have another appearance, here given by

$$
\begin{aligned}
& \mathbf{A}(\mathbf{0})=\left(\begin{array}{ccc}
1-\frac{\tau}{n} w_{o}^{(2,0)} & -\frac{\tau}{n} w_{o}^{(1,1)} & 0 \\
-\frac{\tau}{n} w_{o}^{(1,1)} & 1-\frac{\tau}{n} w_{o}^{(0,2)} & 0 \\
0 & 0 & -1
\end{array}\right) \Rightarrow \mathbf{A}(\mathbf{0})^{-1} \\
& =\left(\begin{array}{ccc}
\gamma\left(\begin{array}{cc}
1-\frac{\tau}{n} w_{o}^{(0,2)} & \frac{\tau}{n} w_{o}^{(1,1)} \\
\frac{\tau}{n} w_{o}^{(1,1)} & 1-\frac{\tau}{n} w_{o}^{(2,0)}
\end{array}\right) & 0 \\
0 & 0 & -1
\end{array}\right),
\end{aligned}
$$

with $\gamma=\frac{-1}{\operatorname{det}(\mathbf{A}(\mathbf{0}))}=\frac{1}{\left.1-(\tau / n) w_{o}^{(2,0)}-\left((\tau / n) w_{o}^{(1,1)}\right)^{2}-(\tau / n) w_{o}^{(0,2)}+(\tau / n)^{2} w_{o}^{(2,0)} w_{o}^{(0,2)}\right)}$, and after inserting Eq. (36) in Eqs. (A7) and (A8), we obtain for the order $k=2$ the solutions

$$
\begin{aligned}
& \mathbf{p}^{(1,0)}(0,0)=\gamma\left(\begin{array}{c}
n\left(n-\tau w_{o}^{(0,2)}\right) \\
n \tau w_{o}^{(1,1)} \\
0
\end{array}\right), \\
& \mathbf{p}^{(0,1)}(0,0)=\gamma\left(\begin{array}{c}
n \tau w_{o}^{(1,1)} \\
n\left(n-\tau w_{o}^{(2,0)}\right) \\
0
\end{array}\right) .
\end{aligned}
$$

For the orders $k \geq 2$, we apply Eq. (A8). The derivatives $\mathbf{A}^{(1,0)}=\left.\frac{d}{d x_{p}} \mathbf{A}\left(\mathbf{p}\left(x_{p}, y_{p}\right)\right)\right|_{x_{p}=0, y_{p}=0}$, etc., are directly obtained by total derivative of Eq. (37) with respect to $x_{p}$ and $y_{p}$, evaluated for $x_{p}=0$ and $y_{p}=0$. For the orders $k=k_{x}+k_{y} \geq 2$, only the results $w_{p}^{\left(k_{x}, k_{y}\right)}(0,0)$ for the propagated wavefront are interesting; therefore, we directly provide those results.

The resulting second-order law is [omitting the argument “(0)"]

$$
\begin{aligned}
& w_{p}^{(2,0)}=\gamma\left(\frac{\tau}{n}\left(w_{o}^{(1,1)}\right)^{2}+\left(1-\frac{\tau}{n} w_{o}^{(0,2)}\right) w_{o}^{(2,0)}\right), \\
& w_{p}^{(1,1)}=\gamma w_{o}^{(1,1)}, \\
& w_{p}^{(0,2)}=\gamma\left(\frac{\tau}{n}\left(w_{o}^{(1,1)}\right)^{2}+\left(1-\frac{\tau}{n} w_{o}^{(2,0)}\right) w_{o}^{(0,2)}\right),
\end{aligned}
$$

which is well known as the propagation equation and is revealed to be a special case of the results. If the coordinate axes coincide with the directions of principal curvature of the wavefront, which means that $w_{o}^{(1,1)}=0$, Eq. (40) can be simplified to

$$
\begin{aligned}
& w_{p}^{(2,0)}=\frac{1}{1-\frac{\tau}{n} w_{o}^{(2,0)}} w_{o}^{(2,0)}, \quad w_{p}^{(1,1)}=0, \\
& w_{p}^{(0,2)}=\frac{1}{1-\frac{\tau}{n} w_{o}^{(0,2)}} w_{o}^{(0,2)} .
\end{aligned}
$$

The resulting higher-order laws can be written in a similar fashion:

$$
\begin{aligned}
& w_{p}^{(3,0)}=\gamma^{3}\left(\left(1-\frac{\tau}{n} w_{o}^{(0,2)}\right)^{3} w_{o}^{(3,0)}+\frac{\tau}{n} w_{o}^{(1,1)}\left(3\left(1-\frac{\tau}{n} w_{o}^{(0,2)}\right)^{2} w_{o}^{(2,1)}\right.\right. \\
& \left.\left.+\frac{\tau}{n} w_{o}^{(1,1)}\left(\frac{\tau}{n} w_{o}^{(0,3)} w_{o}^{(1,1)}\right)+3\left(1-\frac{\tau}{n} w_{o}^{(0,2)}\right)^{2} w_{o}^{(1,2)}\right)\right), \\
& w_{p}^{(2,1)}=\gamma^{3}\left(w_{o}^{(2,1)}+\frac{\tau}{n}\left(w_{o}^{(1,1)}\left(2 w_{o}^{(1,2)}+w_{o}^{(3,0)}\right)\right.\right. \\
& \left.-\left(2 w_{o}^{(0,2)}+w_{o}^{(2,0)}\right) w_{o}^{(2,1)}\right)+\left(\frac{\tau}{n}\right)^{2}\left(w_{o}^{(2,1)} w_{o}^{(0,2)^{2}}\right. \\
& -2\left(w_{o}^{(1,1)}\left(w_{o}^{(1,2)}+w_{o}^{(3,0)}\right)-w_{o}^{(2,0)} w_{o}^{(2,1)}\right) w_{o}^{(0,2)} \\
& \left.+w_{o}^{(0,3)} w_{o}^{(1,1)^{2}}+2 w_{o}^{(1,1)}\left(w_{o}^{(1,1)} w_{o}^{(2,1)}-w_{o}^{(1,2)} w_{o}^{(2,0)}\right)\right) \\
& +\left(\frac{\tau}{n}\right)^{3}\left(w_{o}^{(1,2)} w_{o}^{(1,1)^{3}}-\left(w_{o}^{(0,3)} w_{o}^{(2,0)}+2 w_{o}^{(0,2)} w_{o}^{(2,1)}\right) w_{o}^{(1,1)^{2}}\right. \\
& +w_{o}^{(0,2)}\left(2 w_{o}^{(1,2)} w_{o}^{(2,0)}+w_{o}^{(0,2)} w_{o}^{(3,0)}\right) w_{o}^{(1,1)} \\
& \left.\left.-w_{o}^{(0,2)^{2}} w_{o}^{(2,0)} w_{o}^{(2,1)}\right)\right) \\
& w_{p}^{(1,2)}=\gamma^{3}\left(w_{o}^{(1,2)}+\frac{\tau}{n}\left(w_{o}^{(1,1)}\left(2 w_{o}^{(2,1)}+w_{o}^{(0,3)}\right)\right.\right. \\
& \left.-\left(2 w_{o}^{(2,0)}+w_{o}^{(0,2)}\right) w_{o}^{(1,2)}\right) \\
& +\left(\frac{\tau}{n}\right)^{2}\left(w_{o}^{(1,2)} w_{o}^{(2,0)^{2}}-2\left(w_{o}^{(1,1)}\left(w_{o}^{(2,1)}+w_{o}^{(0,3)}\right)\right.\right. \\
& \left.-w_{o}^{(0,2)} w_{o}^{(1,2)}\right) w_{o}^{(2,0)}+w_{o}^{(3,0)} w_{o}^{(1,1)^{2}} \\
& \left.+2 w_{o}^{(1,1)}\left(w_{o}^{(1,1)} w_{o}^{(1,2)}-w_{o}^{(2,1)} w_{o}^{(0,2)}\right)\right) \\
& +\left(\frac{\tau}{n}\right)^{3}\left(w_{o}^{(2,1)} w_{o}^{(1,1)^{3}}-\left(w_{o}^{(3,0)} w_{o}^{(0,2)}+2 w_{o}^{(2,0)} w_{o}^{(1,2)}\right) w_{o}^{(1,1)^{2}}\right. \\
& +w_{o}^{(2,0)}\left(2 w_{o}^{(2,1)} w_{o}^{(0,2)}+w_{o}^{(2,0)} w_{o}^{(0,3)}\right) w_{o}^{(1,1)} \\
& \left.\left.-w_{o}^{(2,0)^{2}} w_{o}^{(0,2)} w_{o}^{(1,2)}\right)\right) \text {, } \\
& w_{p}^{(0,3)}=\gamma^{3}\left(\left(1-\frac{\tau}{n} w_{o}^{(2,0)}\right)^{3} w_{o}^{(0,3)}+\frac{\tau}{n} w_{o}^{(1,1)}\left(3\left(1-\frac{\tau}{n} w_{o}^{(2,0)}\right)^{2} w_{o}^{(1,2)}\right.\right. \\
& \left.\left.+\frac{\tau}{n} w_{o}^{(1,1)}\left(\frac{\tau}{n} w_{o}^{(3,0)} w_{o}^{(1,1)}\right)+3\left(1-\frac{\tau}{n} w_{o}^{(2,0)}\right)^{2} w_{o}^{(2,1)}\right)\right) \text {. }
\end{aligned}
$$

Equations (40)-(42) show that the result for $w_{p}^{(i, j)}$ can be derived from the result for $w_{p}^{(j, i)}$ by interchanging $i$ and $j$.

\section{Special Case}

Analogous to the special situation that leads to Eq. (30) in the $2 \mathrm{D}$ case, it is possible to find a corresponding special case in the $3 \mathrm{D}$ case. By similar reasoning to the $2 \mathrm{D}$ case and as in [3] , it is found that if all lower order aberrations for $j_{x}+j_{y}<k_{x}+$ $k_{y}$ vanish, then Eq. (A8) will reduce to the lowest term, 
yielding

$$
\begin{aligned}
\mathbf{p}^{\left(k_{x}, 0\right)}= & -\mathbf{A}^{-1} \mathbf{A}^{\left(k_{x}-1,0\right)} \mathbf{A}^{-1} \mathbf{b}_{x}, \quad k_{x} \neq 3, k_{y}=0, \\
\mathbf{p}^{(3,0)}= & \mathbf{A}^{-1}\left(2 \mathbf{A}^{(1,0)} \mathbf{A}^{-1} \mathbf{A}^{(1,0)}-\mathbf{A}^{(2,0)}\right) \mathbf{A}^{-1} \mathbf{b}_{x}, \quad k_{x}=3, k_{y}=0, \\
\mathbf{p}^{(2,1)}= & \mathbf{A}^{-1}\left(\mathbf{A}^{(1,0)} \mathbf{A}^{-1} \mathbf{A}^{(0,1)}\right. \\
& \left.+\mathbf{A}^{(0,1)} \mathbf{A}^{-1} \mathbf{A}^{(1,0)}-\mathbf{A}^{(2,0)}\right) \mathbf{A}^{-1} \mathbf{b}_{x}, \quad k_{x}=2, k_{y}=1, \\
\mathbf{p}^{\left(k_{x}, k_{y}\right)}= & -\mathbf{A}^{-1} \mathbf{A}^{\left(k_{x}-1, k_{y}\right)} \mathbf{A}^{-1} \mathbf{b}_{x}, \quad k_{x} \neq 0, k_{y} \neq 0, k_{x}+k_{y} \neq 3, \\
= & -\mathbf{A}^{-1} \mathbf{A}^{\left(k_{x}, k_{y}-1\right)} \mathbf{A}^{-1} \mathbf{b}_{y}, \\
\mathbf{p}^{(1,2)}= & \mathbf{A}^{-1}\left(\mathbf{A}^{(0,1)} \mathbf{A}^{-1} \mathbf{A}^{(1,0)}\right. \\
& \left.+\mathbf{A}^{(1,0)} \mathbf{A}^{-1} \mathbf{A}^{(0,1)}-\mathbf{A}^{(0,2)}\right) \mathbf{A}^{-1} \mathbf{b}_{y}, \quad k_{x}=1, k_{y}=2, \\
\mathbf{p}^{(0,3)}= & \mathbf{A}^{-1}\left(2 \mathbf{A}^{(0,1)} \mathbf{A}^{-1} \mathbf{A}^{(0,1)}-\mathbf{A}^{(0,2)}\right) \mathbf{A}^{-1} \mathbf{b}_{y}, \quad k_{x}=0, k_{y}=3, \\
\mathbf{p}^{\left(0, k_{y}\right)=} & -\mathbf{A}^{-1} \mathbf{A}^{\left(0, k_{y}-1\right)} \mathbf{A}^{-1} \mathbf{b}_{y}, \quad k_{x}=0, k_{y} \neq 3 .
\end{aligned}
$$

The result in Eq. (43) is similar to Eq. (58) in [3], but it differs due to different conditions under which the matrix A or one of its derivatives vanishes. For finally evaluating Eq. (43), we need the partial derivatives of the matrix $\mathbf{A}$ under the assumption that all lower order aberrations for $j_{x}+j_{y}<k_{x}+k_{y}$ vanish, which is given as

\section{RESULTS}

\section{A. 2D Case}

Equation (22) holds likewise for the derivatives and for the coefficients $a_{o, k}$, and $a_{p, k}$ due to Eqs. (ㅁ) and (ㅁ). In terms of local aberrations, and substituting $\bar{d}=\frac{\tau}{n}$ and $\beta=\frac{1}{1-\frac{\tau}{n} w_{o}^{(2)}}=\frac{1}{1-\frac{d}{n} S_{o}}$, Eq. (22) reads

$$
\begin{aligned}
S_{p}= & \beta S_{o}, \quad E_{p, 3}=\beta^{3} E_{o, 3}, \\
E_{p, 4}= & \beta^{4}\left(E_{o, 4}+3 \frac{d}{n}\left(\beta E_{o, 3}^{2}-\frac{S_{o}^{4}}{n^{2}}\right)\right), \\
E_{p, 5}= & \beta^{5}\left(E_{o, 5}+5 \beta \frac{d}{n} E_{o, 3}\left(2 E_{o, 4}+3 \beta \frac{d}{n} E_{o, 3}^{2}-6 \frac{S_{o}^{3}}{n^{2}}\right)\right), \\
E_{p, 6}= & \beta^{6}\left(E_{o, 6}\right. \\
& +5 \beta \frac{d}{n}\left(3 E_{o, 3} E_{o, 5}+21 \beta \frac{d}{n} E_{o, 3}^{2} E_{o, 4}-12 \frac{S_{o}^{3} E_{o, 4}}{n^{2}}+2 E_{o, 4}^{2}\right. \\
& \left.\left.-9 \beta S_{0}^{2} E_{o, 3}^{2} \frac{3+4 \frac{d}{n} S_{o}}{n^{2}}+21\left(\beta \frac{d}{n}\right)^{2} E_{o, 3}^{4}+9 S_{o}^{6} \frac{1+\frac{d}{n} S_{o}}{n^{4}}\right)\right) .
\end{aligned}
$$

Equation (느) can be generalized for $2<k \leq 6$ to

$$
E_{p, k}=\beta^{k}\left(E_{o, k}+R_{k}\right),
$$

where in $R_{k}$ all wavefront derivatives $E_{o j}$ of lower order $(j<k)$ are expressed in terms of local aberrations.

If only aberrations for one single given order $k$ are present while for all lower orders $j<k$ we have $E_{o, j}=0$, then $\beta=1$

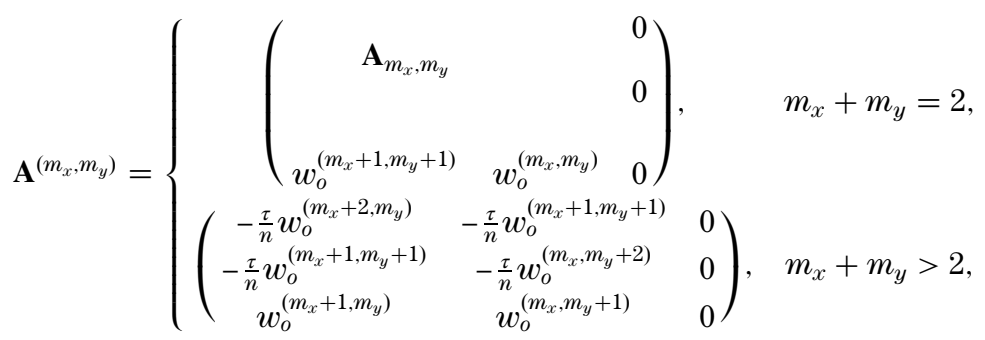

with

$$
\mathbf{A}_{m_{x}, m_{y}}=-\frac{\tau}{n}\left(\begin{array}{cc}
\frac{\tau}{n} \mathbf{v}\left(\begin{array}{c}
w_{o}^{(3,0)} \\
w_{o}^{(2,1)}
\end{array}\right)+w_{o}^{\left(m_{x}+2, m_{y}\right)} & \frac{\tau}{n} \mathbf{v}\left(\begin{array}{c}
w_{o}^{(2,1)} \\
w_{o}^{(1,2)}
\end{array}\right)+w_{o}^{\left(m_{x}+1, m_{y}+1\right)} \\
\frac{\tau}{n} \mathbf{v}\left(\begin{array}{c}
w_{o}^{(2,1)} \\
w_{o}^{(1,2)}
\end{array}\right)+w_{o}^{\left(m_{x}+1, m_{y}+1\right)} & \frac{\tau}{n} \mathbf{v}\left(\begin{array}{c}
w_{o}^{(1,2)} \\
w_{o}^{(0,3)}
\end{array}\right)+w_{o}^{\left(m_{x}, m_{y}+2\right)}
\end{array}\right), \quad \mathbf{v}=\left(\begin{array}{c}
w_{o}^{\left(m_{x}+1, m_{y}\right)} \\
w_{o}^{\left(m_{x}, m_{y}+1\right)}
\end{array}\right),
$$

where $x_{o}^{(1,0)}, x_{o}^{(0,1)}, y_{o}^{(1,0)}, y_{o}^{(0,1)}$, etc., have been substituted by their solutions according to Eq. (39). Inserting $\mathbf{A}^{\left(m_{x}, m_{y}\right)}$ from Eq. (44) and $\mathbf{A}(\mathbf{0})^{-1}$ from Eq. (38) into Eq. (43) yields one common relation for $w_{p}^{\left(k_{x}, k_{y}\right)}$ for the various subcases in Eq. (43) [omitting the argument " $(0,0)$ ”]:

$$
w_{p}^{\left(k_{x}, k_{y}\right)}=w_{o}^{\left(k_{x}, k_{y}\right)}
$$

for all orders $k=k_{x}+k_{y}>2$. and $R_{k}=0$, which means that for fixed order $k$ Eq. (47) will be valid for the vanishing remainder term and the aberration of the propagated wavefront will be equal to the aberration of the original wavefront, independent of the propagation distance $d$ :

$$
E_{p, k}=E_{o, k}
$$




\section{B. 3D Case}

Equation (40) can be summarized as a vector equation in terms of local aberrations and substituting $d=\frac{\tau}{n}$ :

$$
\mathbf{s}_{p}=\gamma\left(\mathbf{s}_{o}+\frac{d}{n}\left(\begin{array}{c}
S_{o, x y}^{2}-S_{o, x x} S_{o, y y} \\
0 \\
S_{o, x y}^{2}-S_{o, x x} S_{o, y y}
\end{array}\right)\right)
$$

with

$$
\gamma=\frac{1}{1-\frac{d}{n} S_{o, x x}-\left(\frac{d}{n} S_{o, x y}\right)^{2}-\frac{d}{n} S_{o, y y}+\left(\frac{d}{n}\right)^{2} S_{o, x x} S_{o, y y}}
$$

also shown in terms of local aberrations.

The vector equation [Eq. (49)] is identical to the well known propagation matrix of Eq. (3). Equivalently, Eq. (42) can be transformed to a vector equation in terms of local aberrations:

$$
{ }^{u} \mathbf{e}_{p, 3}=\left(\begin{array}{cccc}
\beta_{x x}^{3} & 3 \beta_{x x}^{2} \beta_{x y} & 3 \beta_{x x} \beta_{x y}^{2} & \beta_{x y}^{3} \\
\beta_{x x}^{2} \beta_{x y} & \beta_{x x}\left(\beta_{x x} \beta_{y y}+2 \beta_{x y}^{2}\right) & \beta_{x y}\left(2 \beta_{x x} \beta_{y y}+\beta_{x y}^{2}\right) & \beta_{x y}^{2} \beta_{y y} \\
\beta_{x x} \beta_{x y}^{2} & \beta_{x y}\left(2 \beta_{x x} \beta_{y y}+\beta_{x y}^{2}\right) & \beta_{y y}\left(\beta_{x x} \beta_{y y}+2 \beta_{x y}^{2}\right) & \beta_{x y} \beta_{y y}^{2} \\
\beta_{x y}^{3} & 3 \beta_{x y}^{2} \beta_{y y} & 3 \beta_{x y} \beta_{y y}^{2} & \beta_{y y}^{3}
\end{array}\right) \mathbf{e}_{o, 3},
$$

with

$$
\begin{aligned}
\left(\begin{array}{ll}
\beta_{x x} & \beta_{x y} \\
\beta_{x y} & \beta_{y y}
\end{array}\right) & =\left(\mathbf{1}-\frac{\tau}{n}\left(\begin{array}{ll}
w_{o}^{(2,0)} & w_{o}^{(1,1)} \\
w_{o}^{(1,1)} & w_{o}^{(0,2)}
\end{array}\right)\right)^{-1} \\
& =\left(\mathbf{1}-\frac{d}{n}\left(\begin{array}{ll}
S_{o, x x} & S_{o, x y} \\
S_{o, x y} & S_{o, y y}
\end{array}\right)\right)^{-1} .
\end{aligned}
$$

If the coordinate system is chosen in such a way that the $x$ and $y$ axes coincide with the directions of principal curvature of the wavefront, then the equations can be simplified. To do so, the coefficients $a$ of the original wavefront have to be rotated around the axis $\alpha$ of the wavefront (the direction of one principal curvature) with

$$
\alpha=\frac{1}{2} \arctan \left(\frac{-2 S_{o, x y}}{S_{o, y y}-S_{o, x x}}\right) .
$$

Then Eqs. (노) and (50) can be simplified to

$$
\mathbf{s}_{p}=\left(\begin{array}{ccc}
\beta_{x x} & 0 & 0 \\
0 & 0 & 0 \\
0 & 0 & \beta_{y y}
\end{array}\right) \mathbf{s}_{o}
$$

$$
\mathbf{e}_{p, 3}=\left(\begin{array}{cccc}
\beta_{x x}^{3} & 0 & 0 & 0 \\
0 & \beta_{x x}^{2} \beta_{y y} & 0 & 0 \\
0 & 0 & \beta_{x x} \beta_{y y}^{2} & 0 \\
0 & 0 & 0 & \beta_{y y}^{3}
\end{array}\right) \mathbf{e}_{o, 3},
$$

and also, for the radial order $k=4$ an appealing equation can be derived:

$$
\begin{aligned}
& \mathbf{e}_{p, 4}=\left(\begin{array}{ccccc}
\beta_{x x}^{4} & \ldots & & \ldots & 0 \\
\vdots & \beta_{x x}^{3} \beta_{y y}^{1} & & & \vdots \\
& & \beta_{x x}^{2} \beta_{y y}^{2} & & \\
\vdots & & & \beta_{x x}^{1} \beta_{y y}^{3} & \vdots \\
0 & & & \ldots & \beta_{y y}^{4}
\end{array}\right) \text {, }
\end{aligned}
$$

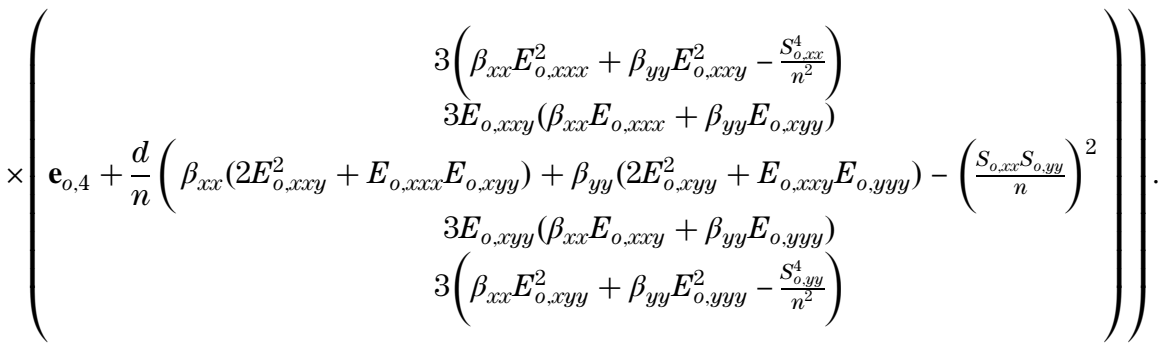


Table 1. Zernike Coefficients of the Original and Propagated Wavefront in Example A1 ${ }^{a}$

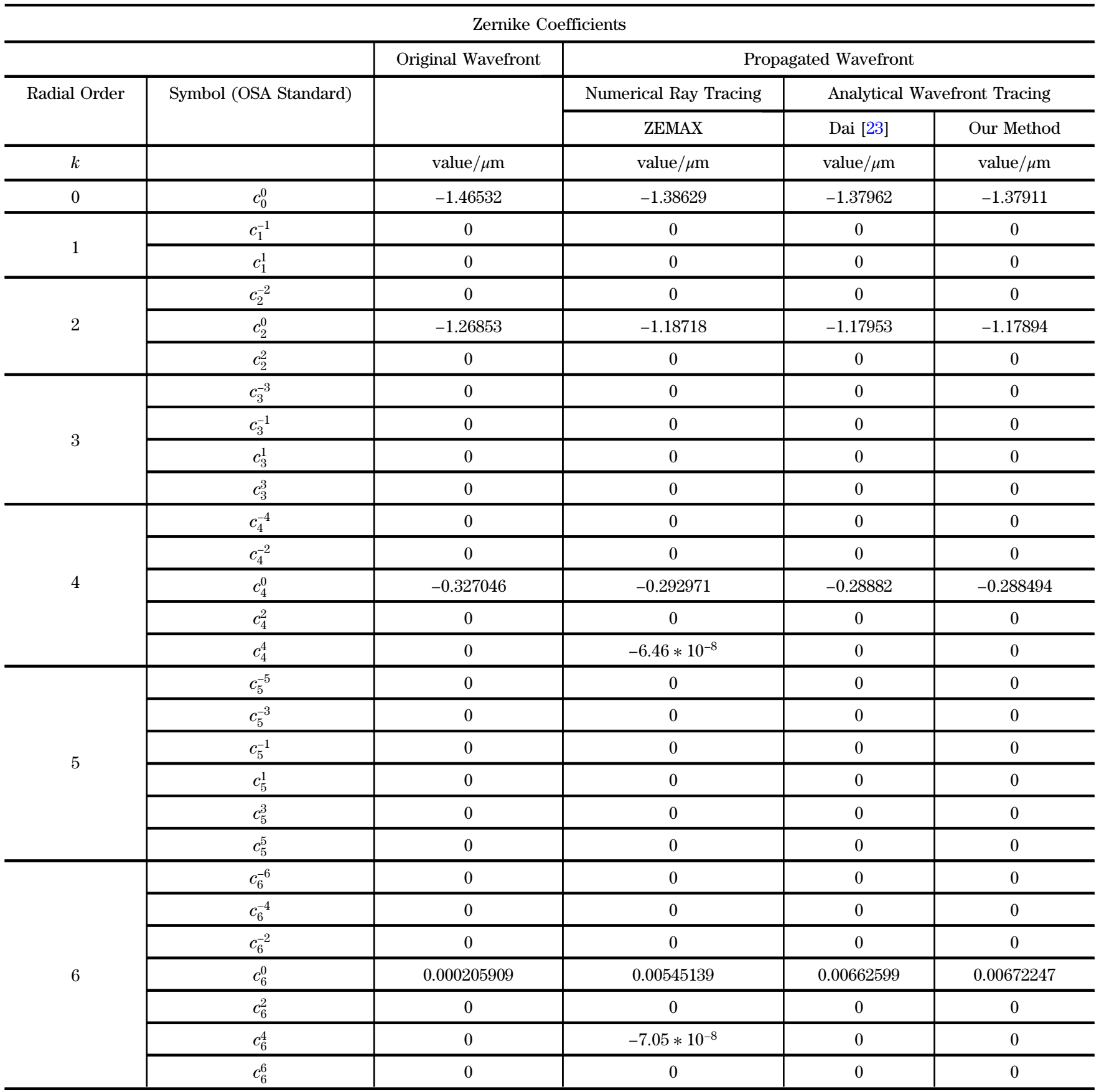

${ }^{a}$ Propagated wavefront, left column: values based on ray tracing (ZEMAX); middle column: values based on method described in [14 $\left.\underline{23}\right]$; right column: values based on our method.

Afterward, the coefficients of the propagated wavefront have to be rerotated to the original coordinate system. The resulting coefficients are then, of course, identical to the coefficients calculated by Eqs. (49) and (50).

Equations (53) and (54) can be generalized for $2<k \leq 6$ to

$$
\mathbf{e}_{p, k}=\mathbf{B}_{k}\left(\mathbf{e}_{o, k}+\mathbf{r}_{k}\right),
$$

where $\mathbf{r}_{k}$ is a vector collecting the remainder terms $R_{k_{x}, k_{y}}$ analogously to $R_{k}$ in Eq. (47), and with

$$
\mathbf{B}_{k}=\left(\begin{array}{ccccc}
\beta_{x x}^{k} & \ldots & & \cdots & 0 \\
\vdots & \beta_{x x}^{k-1} \beta_{y y}^{1} & & & \vdots \\
& & \ddots & & \\
\vdots & & & \beta_{x x}^{1} \beta_{y y}^{k-1} & \vdots \\
0 & & & \cdots & \beta_{y y}^{k}
\end{array}\right) .
$$

The result of the special case treated in Eqs. (느)-(ㄴ5) can be summarized, in a similar fashion to Eq. (55), to a vector equation in the very appealing form 
Table 2. Zernike Coefficients of the Original and Propagated Wavefront in Example A2 ${ }^{a}$

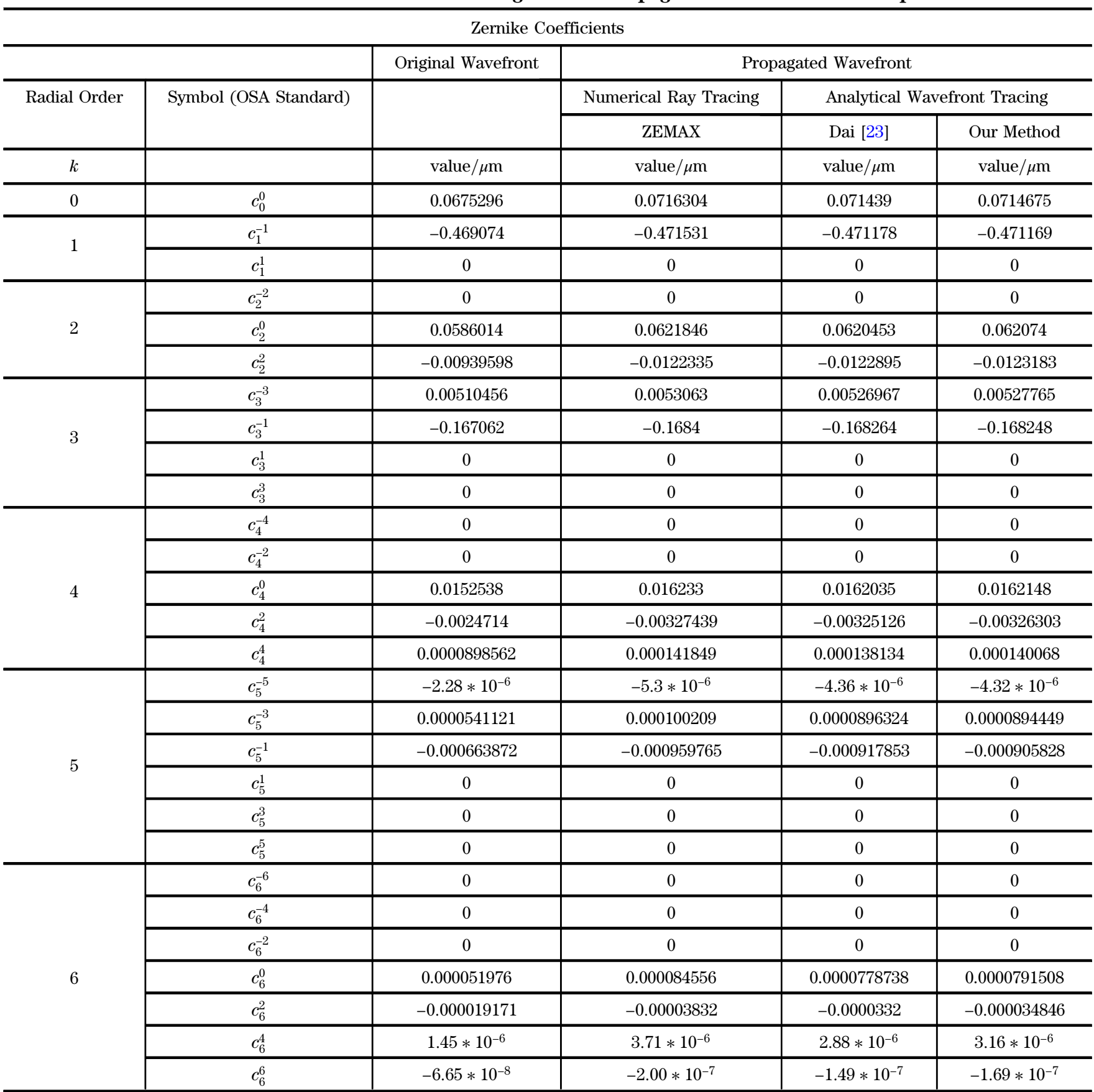

\footnotetext{
${ }^{a}$ Propagated wavefront, left column: values based on ray tracing (ZEMAX); middle column: values based on method described in [프, $\left.\underline{23}\right]$; right column: values based
} on our method.

$$
\mathbf{e}_{p, k}=\mathbf{e}_{o, k},
$$

which is Eq. (55) for $\mathbf{r}_{k}=\mathbf{0}$. Equation (57), an interesting result of the present paper, is the propagation equation for aberrations of fixed order $k \geq 3$ under the assumption that all aberrations with an order lower than $k$ vanish, which means that the aberration of the propagated wavefront will be equal to the aberration of the original wavefront, independent of the propagation distance $d$.

\section{EXAMPLES AND APPLICATIONS}

One important application of the derived equations is that they allow us to determine the aberrations of a wavefront by propagation, which not only has a defined power $S_{o}$ but also shows aberrations of higher aberrations. Because of the analytical nature of the equations it is not necessary to use an iterative numerical method.

We use the derived equations [Eqs. (52)-(54)] to determine the aberrations of the propagated wavefront up to the radial order $k=6$ and compare them first with the results calculated by the analytical wavefront approach described by Dai [14] and Dai et al. [23]. One approximation with significant influence of the analytical wavefront approach described by Dai $e t$ $a l$. is that the transformation of the coefficients was solved without simultaneously solving the coordinate dependence. 
Table 3. Zernike Coefficients of the Original and Propagated Wavefront in Example B1 ${ }^{a}$

\begin{tabular}{|c|c|c|c|c|c|}
\hline \multicolumn{6}{|c|}{ Zernike Coefficients } \\
\hline & & \multirow[t]{3}{*}{ Original Wavefront } & \multicolumn{3}{|c|}{ Propagated Wavefront } \\
\hline \multirow[t]{2}{*}{ Radial Order } & \multirow[t]{2}{*}{ Symbol (OSA Standard) } & & \multirow{2}{*}{$\frac{\text { Numerical Ray Tracing }}{\text { ZEMAX }}$} & \multicolumn{2}{|c|}{ Analytical Wavefront Tracing } \\
\hline & & & & Dai [를 & Our Method \\
\hline$k$ & & value $/ \mu \mathrm{m}$ & value $/ \mu \mathrm{m}$ & value $/ \mu \mathrm{m}$ & value $/ \mu \mathrm{m}$ \\
\hline 0 & $c_{0}^{0}$ & -50.1362 & -34.3311 & -26.5342 & -34.3309 \\
\hline \multirow{2}{*}{1} & $c_{1}^{-1}$ & 0 & $1.18 * 10^{-8}$ & 0 & 0 \\
\hline & $c_{1}^{1}$ & 0 & $1.18 * 10^{-8}$ & 0 & 0 \\
\hline \multirow{3}{*}{2} & $c_{2}^{-2}$ & 0 & $-1.76 * 10^{-8}$ & 0 & 0 \\
\hline & $c_{2}^{0}$ & -29.3453 & -19.9117 & -14.9908 & -19.9115 \\
\hline & $c_{2}^{2}$ & 0 & 0 & 0 & 0 \\
\hline \multirow{4}{*}{3} & $c_{3}^{-3}$ & 0 & $5.88 * 10^{-9}$ & 0 & 0 \\
\hline & $c_{3}^{-1}$ & 0 & 0 & 0 & 0 \\
\hline & $c_{3}^{1}$ & 0 & 0 & 0 & 0 \\
\hline & $c_{3}^{3}$ & 0 & $-5.88 * 10^{-9}$ & 0 & 0 \\
\hline \multirow{5}{*}{4} & $c_{4}^{-4}$ & 0 & 0 & 0 & 0 \\
\hline & $c_{4}^{-2}$ & 0 & 0 & 0 & 0 \\
\hline & $c_{4}^{0}$ & -0.309331 & -0.069658 & 0.261713 & -0.0695334 \\
\hline & $c_{4}^{2}$ & 0 & 0 & 0 & 0 \\
\hline & $c_{4}^{4}$ & 0 & $-1.76 * 10^{-8}$ & 0 & 0 \\
\hline \multirow{6}{*}{5} & $c_{5}^{-5}$ & 0 & $1.76 * 10^{-8}$ & 0 & 0 \\
\hline & $c_{5}^{-3}$ & 0 & 0 & 0 & 0 \\
\hline & $c_{5}^{-1}$ & 0 & 0 & 0 & 0 \\
\hline & $c_{5}^{1}$ & 0 & 0 & 0 & 0 \\
\hline & $c_{5}^{3}$ & 0 & 0 & 0 & 0 \\
\hline & $c_{5}^{5}$ & 0 & $1.76 * 10^{-8}$ & 0 & 0 \\
\hline \multirow{7}{*}{6} & $c_{6}^{-6}$ & 0 & $2.94 * 10^{-8}$ & 0 & 0 \\
\hline & $c_{6}^{-4}$ & 0 & $-1.18 * 10^{-8}$ & 0 & 0 \\
\hline & $c_{6}^{-2}$ & 0 & 0 & 0 & 0 \\
\hline & $c_{6}^{0}$ & -0.000110975 & 0.000437634 & 0.00598868 & 0.000473857 \\
\hline & $c_{6}^{2}$ & 0 & 0 & 0 & 0 \\
\hline & $c_{6}^{4}$ & 0 & $1.18 * 10^{-8}$ & 0 & 0 \\
\hline & $c_{6}^{6}$ & 0 & 0 & 0 & 0 \\
\hline
\end{tabular}

${ }^{a}$ Propagated wavefront, left column: values based on ray tracing (ZEMAX); middle column: values based on method described in [14,23]; right column: values based on our method.

As we show in the examples, and as is also stated in [23], it is absolutely necessary to solve both dependencies simultaneously if wavefronts contain both low-order and high-order aberrations.

Second, we compare our results with the results calculated by a numerical ray-tracing approach using the optical design package ZEMAX, followed by a Zernike analysis.

We would like to stress again that our local aberration values are obtained by an analytical method and therefore by definition are exact. The transformation of our local Taylor coefficients to Zernike coefficients, on the other hand, yields only an (however, very good) approximation for their numerical values based on the assumption that the truncated subspaces of order $k=6$ describe the aberrations sufficiently well. Still, within this approximation, the results are analytical, such that a Zernike coefficient obtained as zero is exactly zero, whereas a ray-tracing value is always numerical by its nature, resulting in small deviations from zero (Tables $1-4$ ).

The necessary transformation between Zernike and Taylor coefficients, itself being state of the art [27,28], is in our case also accompanied by the transformation from an OPD wave aberration to a wavefront aberration referring to the sagitta, 
Table 4. Zernike Coefficients of the Original and Propagated Wavefront in Example B2 ${ }^{a}$

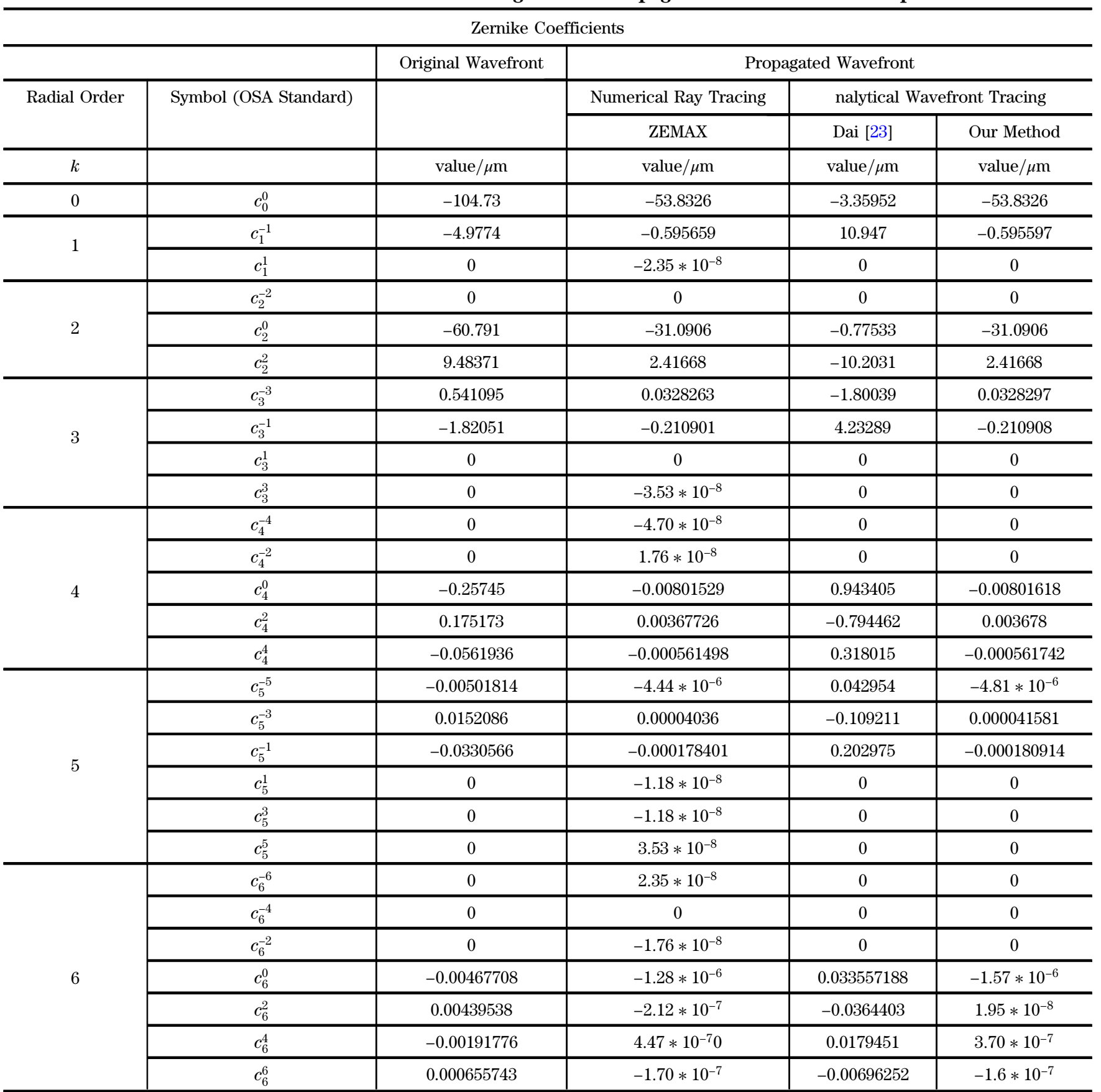

\footnotetext{
${ }^{a}$ Propagated wavefront, left column: values based on ray tracing (ZEMAX); middle column: values based on method described in [느,23]; right column: values based
} on our method.

which is discussed in detail in [3]. The logical flow of the transformations is illustrated in Fig. $\underline{3}$.

Examples A1 and A2 are characterized by the specific feature that the first and second derivatives are zero, which means that the coefficients of Taylor monomials of first and second order are also zero (see Table 5). This implies that the low-order aberrations (LOA) (radial order $k<3$ ) expressed as Taylor monomials are zero, while in examples B1 and B2 low-order and high-order aberrations do occur (see Table 6). In examples A1 and B1 only rotationally symmetric aberrations are present, while in examples A2 and B2 non-rotationally symmetric aberrations like coma, trefoil, and secondary astigmatism also occur.

The value of the propagation distance $d$ is $20 \mathrm{~mm}$, the pupil diameter $d_{0}$ is $6 \mathrm{~mm}$ and the refractive index $n$ is 1 in all four examples.

For giving some more insight about how the resulting values are obtained within our framework, we provide explicit formulas for the Taylor coefficients in the case of the rotationally symmetric examples A1 and B1. In this case all the odd order coefficients vanish, and we directly obtain, for order $n=2$ from Eq. (49), 


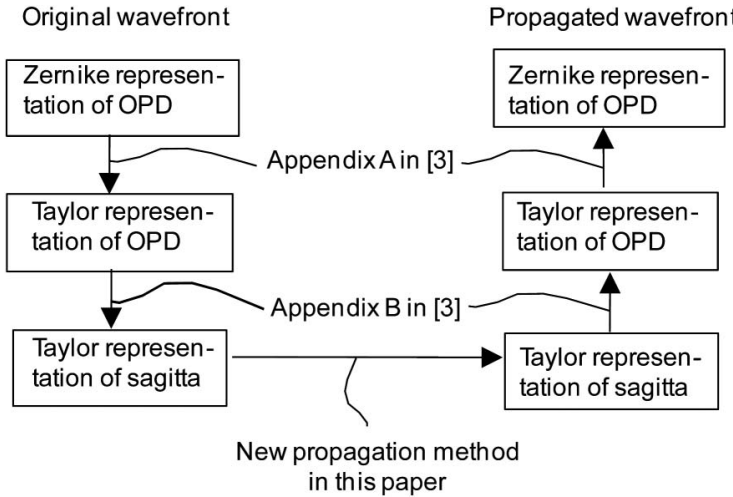

Fig. 3. Logical flow of the computation of the Zernike coefficients of a propagated wavefront for the given Zernike coefficients of the original wavefront.

$$
\mathbf{s}_{p}=\left(\begin{array}{c}
\beta_{x x x} S_{o, x x} \\
0 \\
\beta_{y y} S_{o, x x}
\end{array}\right) .
$$

For order $n=4$, it follows from Eq. (54) that

$$
\mathbf{e}_{p, 4}=\left(\begin{array}{c}
\beta_{x x}^{4} E_{o, x x x x} \\
0 \\
\beta_{x x}^{2} \beta_{y y}^{2} E_{o, x x y y} \\
0 \\
\beta_{y y}^{4} E_{o, y y y y}
\end{array}\right)-\frac{d}{n^{3}}\left(\begin{array}{c}
3 \beta_{x x}^{4} S_{o, x x}^{4} \\
0 \\
\beta_{x x}^{2} \beta_{y y}^{2}\left(S_{o, x x} S_{o, y y}\right)^{2} \\
0 \\
3 \beta_{y y}^{4} S_{o, y y}^{4}
\end{array}\right),
$$

and for order $n=6$, it follows from the general solution in Eq. (55) after some algebra that

$$
\begin{aligned}
E_{p, x x x x x x}= & \beta_{x x}^{6}\left(E_{o, x x x x x x}+5 \frac{d}{n^{5}}\left(2 \beta _ { x x } \left(n^{2} E_{o, x x x x}\right.\right.\right. \\
& \left.\left.\left.-3 S_{o, x x}^{3}\right)-9 S_{o, x x}^{6}\right)\right), \\
E_{p, x x x x y y}= & \beta_{x x}^{4} \beta_{y y}^{2}\left(E_{o, x x x x y y}+\frac{d}{n^{6}}\left(6 \beta_{y y} n^{5} E_{o, x x x x}^{2}\right.\right. \\
& +\beta_{x x} S_{o, x x} S_{o, y y}^{2}\left(3 S_{o, x x}^{3}\left(n+d\left(3-2 \beta_{y y}\right) S_{o, x x}+2 n \beta_{y y}\right)\right. \\
& \left.-4 n^{3} E_{o, x x x x}\right)+4 n^{3} \beta_{x x} E_{o, x x y y} \\
& \left.\left.\times\left(n^{2} E_{o, x x x x}+3 S_{o, x x}^{3}\left(\left(\beta_{y y}-2\right) S_{o, x x}-\beta_{y y} S_{o, y y}\right)\right)\right)\right), \\
E_{p, x x y y y y}= & E_{p, x x x x y y}(x \leftrightarrow y), \quad E_{p, y y y y y y}=E_{p, x x x x x x x}(x \leftrightarrow y) .
\end{aligned}
$$

Example A1: Given is the original wavefront expressed with Zernike polynomials. The coefficients of the Zernike polynomials are zero except defocus $c_{2}^{0}$, spherical aberration $c_{4}^{0}$, and secondary spherical aberration $c_{6}^{0}$, their values being chosen such that the second-order local aberrations vanish. In this case the equations derived by Dai $e t$ al. are a very good approximation, as stated in the conclusion [23].

The values of the original wavefront and the resulting values of the propagated wavefront derived by all three methods are provided in Table 1 . The agreement between the results of all three methods is obvious. Additionally, we have provided the values of the local aberrations before and after propagation in Table 5 .

Example A2: In this example the original wavefront shows defocus, astigmatism, coma, trefoil, spherical aberration, sec- ondary astigmatism, quadrafoil, secondary coma, secondary trefoil, secondary spherical aberration, secondary quadrafoil, and tertiary astigmatism.

The values of the original wavefront and the resulting values of the propagated wavefront derived by all three methods are provided in Table 2. Also in this more complex case, the agreement between the results of all three methods is obvious. Again, values of local aberrations are provided in Table 5 .

Example B1: This example is equivalent to example $\overline{\mathrm{A}} 1$. Also in this example, the coefficients of the Zernike polynomials are zero except defocus $c_{2}^{0}$, spherical aberration $c_{4}^{0}$, and secondary spherical aberration $c_{6}^{0}$. However, now the original wavefront is characterized by the specific feature that LOA (radial order $k=2$ ) expressed as Taylor monomials are nonzero (see Table 6). In this case the equations derived by Dai $e t a l$. are not a good approximation, as stated in the conclusion [23].

The values of the original wavefront and the resulting values of the propagated wavefront derived by all three methods are provided in Table 3. The agreement between the results derived by the optical design package ZEMAX and our analytical method is obvious, while the results derived by the analytical method of Dai et al. [23] differ strongly. The wrong results derived by the analytical method of Dai et al. are based on the fact that in this method the coordinate change by propagation is not considered. This approximation will lead to the wrong results if low- and high-order aberrations occur simultaneously, as stated by Dai et al. [23] in their conclusion.

Additionally, we have provided the values of the local aberrations before and after propagation in Table 6 .

Example B2: This example is equivalent to example A2, but here the original wavefront is also, as in example B1, characterized by the specific feature that the LOA (radial order $k=2$ ) expressed as Taylor monomials are nonzero (see Table 6). In this case the equations derived by Dai et al. [23] are also not a good approximation.

In contrast to example B1, in example B2 non-rotationally symmetric aberrations such as coma, trefoil, and secondary astigmatism occur.

The values of the original wavefront and the resulting values of the propagated wavefront derived by all three methods are provided in Table 4 . The agreement between the results derived by the optical design package ZEMAX and our analytical method is also, in this more complex example, obvious, while the results derived by the analytical method of Dai et al. [23] differ strongly.

Again, values of local aberrations are provided in Table 6 .

\section{SUMMARY}

The equations in [3] describe the local higher-order aberrations of a local wavefront after refraction, which allow us to calculate analytically the HOA of an outgoing wavefront directly from the aberrations of the incoming wavefront and the refractive surface after refraction in a very fast manner.

For propagation all hitherto existing analytical equations are still restricted by some approximations. As is written by Dai et al. [23], further study would be necessary to obtain a unified formulation for wavefronts containing both loworder and high-order aberrations. In the present work we have succeeded in developing such a unified analytical propagation method. 
Table 5. Local Aberrations of the Original and Propagated Wavefront (Values Based on Our Method) in Examples A1 and A2

\begin{tabular}{|c|c|c|c|c|c|}
\hline \multicolumn{6}{|c|}{ Local Aberrations (Our Method) } \\
\hline \multirow[b]{2}{*}{ Radial Order } & \multirow[b]{2}{*}{ Symbol } & \multicolumn{2}{|c|}{ Example A1 } & \multicolumn{2}{|c|}{ Example A2 } \\
\hline & & Original Wavefront & Propagated Wavefront & Original Wavefront & Propagated Wavefront \\
\hline$k$ & & value $* m^{k-1}$ & value $* m^{k-1}$ & value $* m^{k-1}$ & value $* m^{k-1}$ \\
\hline 0 & $E$ & 0 & 0 & 0 & 0 \\
\hline \multirow{2}{*}{1} & $E_{x}$ & 0 & 0 & 0 & 0 \\
\hline & $E_{y}$ & 0 & 0 & 0 & 0 \\
\hline \multirow{3}{*}{2} & $E_{x x}$ & 0 & 0 & 0 & 0 \\
\hline & $E_{x y}$ & 0 & 0 & 0 & 0 \\
\hline & $E_{y y}$ & 0 & 0 & 0 & 0 \\
\hline \multirow{4}{*}{3} & $E_{x x x}$ & 0 & 0 & 0 & 0 \\
\hline & $E_{x x y}$ & 0 & 0 & -99.919 & 99.9191 \\
\hline & $E_{x y y}$ & 0 & 0 & 0 & 0 \\
\hline & $E_{y y y}$ & 0 & 0 & -311.92 & 311.924 \\
\hline \multirow{5}{*}{4} & $E_{x x x x}$ & $-1.3049 \times 10^{6}$ & $-1.3049 \times 10^{6}$ & 50653 & 51253 \\
\hline & $E_{x x x y}$ & 0 & 0 & 0 & 0 \\
\hline & $E_{x x y y}$ & $-4.3498 \times 10^{5}$ & $-4.3498 \times 10^{5}$ & 19729 & 20752 \\
\hline & $E_{x y y y}$ & 0 & 0 & 0 & 0 \\
\hline & $E_{y y y y}$ & $-1.3049 \times 10^{6}$ & $-1.3049 \times 10^{6}$ & 68329 & 74167 \\
\hline \multirow{6}{*}{5} & $E_{x x x x x}$ & 0 & 0 & 0 & 0 \\
\hline & $E_{x x x x y}$ & 0 & 0 & $-1.9975 \times 10^{6}$ & $-2.6475 \times 10^{6}$ \\
\hline & $E_{x x x y y}$ & 0 & 0 & 0 & 0 \\
\hline & $E_{x x y y y}$ & 0 & 0 & $-2.1749 \times 10^{6}$ & $-2.9387 \times 10^{6}$ \\
\hline & $E_{x y y y y}$ & 0 & 0 & 0 & 0 \\
\hline & $E_{\text {yyyyy }}$ & 0 & 0 & $-1.1823 \times 10^{7}$ & $-1.6268 \times 10^{7}$ \\
\hline \multirow{7}{*}{6} & $E_{x x x x x x x}$ & $1.0761 \times 10^{10}$ & $3.5133 \times 10^{11}$ & $1.6856 \times 10^{9}$ & $2.2744 \times 10^{9}$ \\
\hline & $E_{x x x x x y}$ & 0 & 0 & 0 & 0 \\
\hline & $E_{x x x x y y}$ & $2.1522 \times 10^{9}$ & $7.0266 \times 10^{10}$ & $4.6196 \times 10^{8}$ & $6.7583 \times 10^{8}$ \\
\hline & $E_{x x x y y y}$ & 0 & 0 & 0 & 0 \\
\hline & $E_{x x y y y y}$ & $2.1522 \times 10^{9}$ & $7.0266 \times 10^{10}$ & $6.0316 \times 10^{8}$ & $9.3213 \times 10^{8}$ \\
\hline & $E_{x y y y y y}$ & 0 & 0 & 0 & 0 \\
\hline & $E_{\text {yyyyyy }}$ & $1.0761 \times 10^{10}$ & $3.5133 \times 10^{11}$ & $3.8114 \times 10^{9}$ & $6.1388 \times 10^{9}$ \\
\hline
\end{tabular}

These results include, as a special case, the classical scalar vertex correction formula as well as the well-known transfer matrix equation (order $k=2$ ) but extend these propagation equations to aberrations of higher order $(k>2)$.

The propagation equations are relations between the original wavefront and the propagated wavefront. In detail, we have defined local aberrations of those two wavefronts in terms of local power series coefficients, which describe the wavefronts in a general coordinate systems aligned with the chief ray's normal. The general propagation equations are established as a sequence of analytical relations between these series coefficients. We have been able to show that to each given order $k \geq 2$, it is possible to assign one equation taken from that sequence whose leading-order terms represent a straightforward generalization of the transfer equation to the order $k$, and which in general contains some additional terms whose order is always less than $k$. A direct consequence is that if only aberrations of one single order $k(k>2)$ are present, then the aberrations are not changed by propagation, which reads $E_{p, k}=E_{o, k}$ for the 2D problem, and the vector-valued version of which reads $\mathbf{e}_{p, k}=$ $\mathbf{e}_{o, k}$ in the 3D case.

For convenience, we have distinguished between the $2 \mathrm{D}$ and 3D problems in deriving the propagation equations. We have provided the general formalism, and for the orders $k \leq 6$, we have provided explicit formulas for the resulting terms in the $2 \mathrm{D}$ case. 
Table 6. Local Aberrations of the Original and Propagated Wavefront (Values Based on Our Method) in Examples B1 and B2

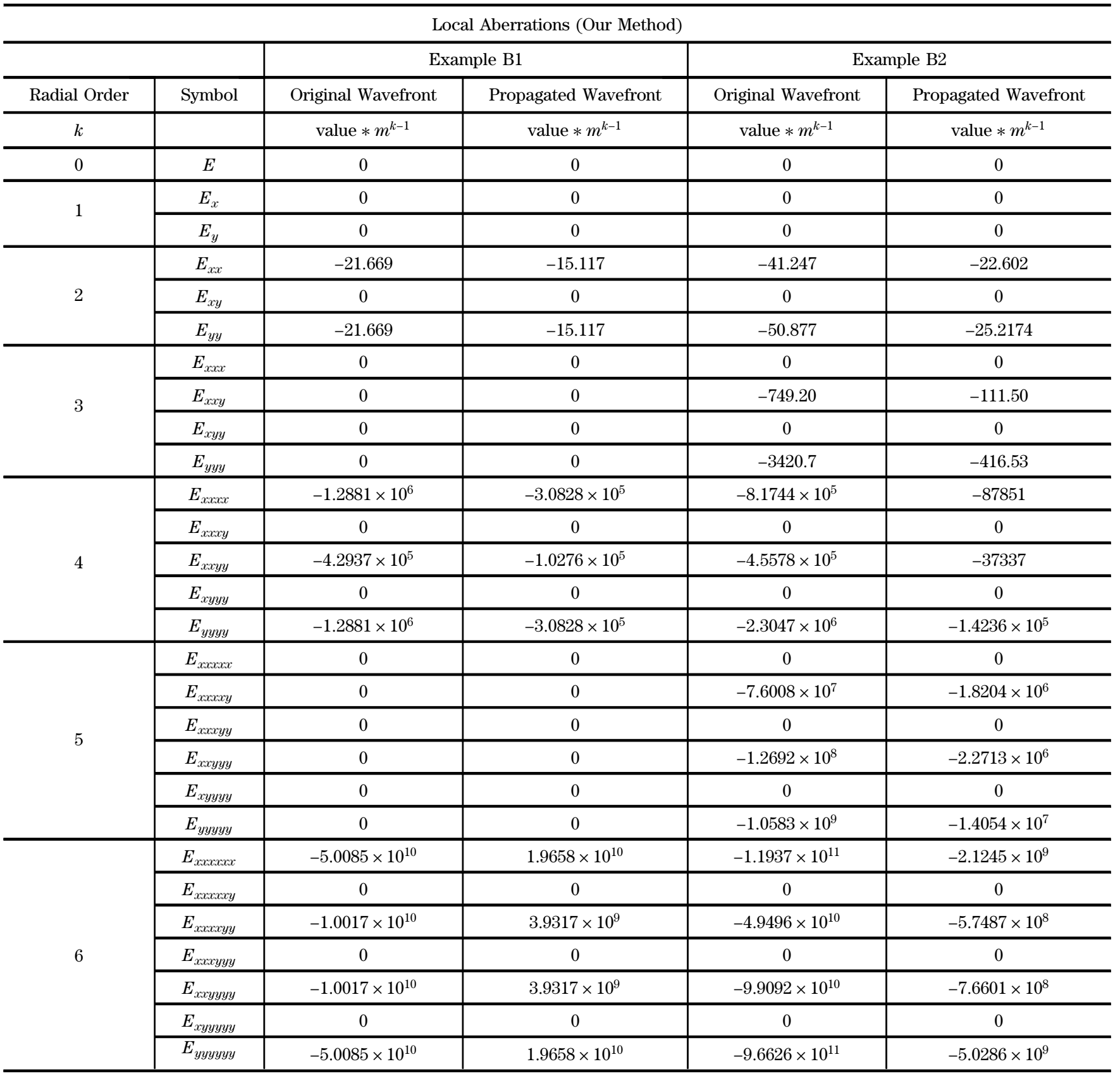

The main advantage of our approach is that it is based exclusively on analytical formulas. This saves much computation time compared to numerical iteration routines, which would otherwise be necessary for determining the higher order aberrations.

With the method developed in this work, it is now possible to calculate the local higher-order aberrations of the propagated wavefront directly in an analytical way from the aberrations of the original wavefront and the propagation distance. Although our method is based on local techniques, it yields results that are by no means restricted to small apertures, as we have shown theoretically as well as in four examples.

\section{APPENDIX A: EQUATIONS DERIVED IN [3]}

$$
\mathbf{n}(v):=\frac{1}{\sqrt{1+v^{2}}}\left(\begin{array}{c}
-v \\
1
\end{array}\right)
$$

$$
\begin{aligned}
& \mathbf{n}(0):=\left(\begin{array}{l}
0 \\
1
\end{array}\right), \quad \mathbf{n}^{(1)}(0):=\left(\begin{array}{c}
-1 \\
0
\end{array}\right), \quad \mathbf{n}^{(2)}(0):=\left(\begin{array}{c}
0 \\
-1
\end{array}\right), \\
& \mathbf{n}^{(3)}(0):=\left(\begin{array}{l}
3 \\
0
\end{array}\right), \quad \mathbf{n}^{(4)}(0):=\left(\begin{array}{l}
0 \\
9
\end{array}\right), \text { etc. }
\end{aligned}
$$




$$
\mathbf{p}^{(1)}\left(y_{p}\right)=\mathbf{A}\left(\mathbf{p}\left(y_{p}\right)\right)^{-1} \mathbf{b} \text {. }
$$

$$
\begin{aligned}
& \mathbf{p}^{(1)}(0)=\mathbf{A}^{-1} \mathbf{b}, \\
& \mathbf{p}^{(2)}(0)=\left(\mathbf{A}^{-1}\right)^{(1)} \mathbf{b}, \ldots, \mathbf{p}^{(k)}(0)=\left(\mathbf{A}^{-1}\right)^{(k-1)} \mathbf{b},
\end{aligned}
$$

where $\quad \mathbf{A}^{-1}=\mathbf{A}(\mathbf{p}(0))^{-1}=\mathbf{A}(\mathbf{0})^{-1} \quad$ and $\quad\left(\mathbf{A}^{-1}\right)^{(1)}=$ $\left.\frac{d}{d y_{p}} \mathbf{A}\left(\mathbf{p}\left(y_{p}\right)\right)^{-1}\right|_{y_{p}=0}, \ldots,\left(\mathbf{A}^{-1}\right)^{(k-1)}=\left.\frac{d^{k-1}}{d y_{p}^{k-1}} \mathbf{A}\left(\mathbf{p}\left(y_{p}\right)\right)^{-1}\right|_{y_{p}=0}$.

$$
\mathbf{A p}^{(1)}(0)=\mathbf{b}
$$

$$
\mathbf{A}^{(1)} \mathbf{p}^{(1)}(0)+\mathbf{A p}^{(2)}(0)=\mathbf{0},
$$

$$
\mathbf{A}^{(2)} \mathbf{p}^{(1)}(0)+2 \mathbf{A}^{(1)} \mathbf{p}^{(2)}(0)+\mathbf{A p}^{(3)}(0)=\mathbf{0} \ldots,
$$

$$
\sum_{j=1}^{k}\left(\begin{array}{l}
k-1 \\
j-1
\end{array}\right) \mathbf{A}^{(k-j)} \mathbf{p}^{(j)}(0)=\mathbf{0}, \quad k \geq 2 .
$$

$$
\begin{aligned}
& \mathbf{p}^{(1)}(0)=\mathbf{A}^{-1} \mathbf{b}, \quad k=1, \\
& \mathbf{p}^{(k)}(0)=-\mathbf{A}^{-1} \sum_{j=1}^{k-1}\left(\begin{array}{c}
k-1 \\
j-1
\end{array}\right) \mathbf{A}^{(k-j)} \mathbf{p}^{(j)}(0), \quad k \geq 2 .
\end{aligned}
$$

$$
\begin{aligned}
& \mathbf{p}^{(1,0)}(0,0)=\mathbf{A}^{-1} \mathbf{b}_{x}, \quad \mathbf{p}^{(0,1)}(0,0)=\mathbf{A}^{-1} \mathbf{b}_{y}, \\
& \mathbf{p}^{(2,0)}(0,0)=\left(\mathbf{A}^{-1}\right)^{(1,0)} \mathbf{b}_{x}, \\
& \mathbf{p}^{(1,1)}(0,0)=\left(\mathbf{A}^{-1}\right)^{(0,1)} \mathbf{b}_{x}=\left(\mathbf{A}^{-1}\right)^{(1,0)} \mathbf{b}_{y}, \\
& \mathbf{p}^{(0,2)}(0,0)=\left(\mathbf{A}^{-1}\right)^{(0,1)} \mathbf{b}_{y} \cdots \\
& \mathbf{p}^{\left(k_{x}, k_{y}\right)}(0,0)= \begin{cases}\left(\mathbf{A}^{-1}\right)^{\left(k_{x}-1,0\right)} \mathbf{b}_{x}, & k_{x} \neq 0, k_{y}=0 \\
\left(\mathbf{A}^{-1}\right)^{\left(k_{x}-1, k_{y}\right)} \mathbf{b}_{x}=\left(\mathbf{A}^{-1}\right)^{\left(k_{x}, k_{y}-1\right)} \mathbf{b}_{y}, & k_{x} \neq 0, k_{y} \neq 0 . \\
\left(\mathbf{A}^{-1}\right)^{\left(0, k_{y}-1\right)} \mathbf{b}_{y}, & k_{x}=0, k_{y} \neq 0\end{cases}
\end{aligned}
$$

$$
\mathbf{p}^{\left(k_{x}, 0\right)}(0,0)=-\mathbf{A}^{-1} \sum_{j_{x}=1}^{k_{x}-1}\left(\begin{array}{c}
k_{x}-1 \\
j_{x}-1
\end{array}\right) \mathbf{A}^{\left(k_{x}-j_{x}, 0\right)} \mathbf{p}^{\left(j_{x}, 0\right)},
$$$$
k_{x} \geq 2, k_{y}=0,
$$

$$
\mathbf{p}^{\left(k_{x}, k_{y}\right)}(0,0)=-\mathbf{A}^{-1} \sum_{\substack{j_{x} \geq 1, j_{y} \geq 0 \\
j_{x}+j_{y}<k_{x}+k_{y}}}\left(\begin{array}{c}
k_{x}-1 \\
j_{x}-1
\end{array}\right)\left(\begin{array}{c}
k_{y} \\
j_{y}
\end{array}\right) \mathbf{A}^{\left(k_{x}-j_{x}, k_{y}-j_{y}\right)} \mathbf{p}^{\left(j_{x} j_{y}\right)},
$$

$$
\begin{aligned}
& =-\mathbf{A}^{-1} \sum_{\substack{j_{x} \geq 0, j_{y} \geq 1 \\
j_{x}+j_{y}<k_{x}+k_{y}}}\left(\begin{array}{c}
k_{x} \\
j_{x}
\end{array}\right)\left(\begin{array}{c}
k_{y}-1 \\
j_{y}-1
\end{array}\right) \mathbf{A}^{\left(k_{x}-j_{x}, k_{y}-j_{y}\right)} \mathbf{p}^{\left(j_{x} j_{y}\right)}, \\
& k_{x} \neq 0, k_{y} \neq 0, \\
& \mathbf{p}^{\left(0, k_{y}\right)}(0,0)=-\mathbf{A}^{-1} \sum_{j_{y}=1}^{k_{y}-1}\left(\begin{array}{c}
k_{y}-1 \\
j_{y}-1
\end{array}\right) \mathbf{A}^{\left(0, k_{y}-j_{y}\right)} \mathbf{p}^{\left(0, j_{y}\right)}, \\
& k_{x}=0, k_{y} \geq 2 .
\end{aligned}
$$

\section{REFERENCES}

1. M. Born and E. Wolf, Principles of Optics (Pergamon, 1980), pp. 109-232.

2. V. Mahajan, Optical Imaging and Aberrations: Part I. Ray Geometrical Optics (SPIE, 1998), pp. 91-361.

3. G. Esser, W. Becken, W. Müller, P. Baumbach, J. Arasa, and D. Uttenweiler, "Derivation of the refractive equations for higher order aberrations of local wavefronts by oblique incidence," J. Opt. Soc. Am. A 27, 218-237 (2010).

4. W. Becken, A. Seidemann, H. Altheimer, G. Esser, and D. Uttenweiler, "Spectacle lenses in sports: optimization of the imaging properties based on physiological aspects," Z. Med Phys. 17, 56-66 (2007).

5. W. Harris, "Wavefronts and their propagation in astigmatic systems," Optom. Vis. Sci. 73, 606-612 (1996).

6. E. Acosta and R. Blendowske, "Paraxial propagation of astigmatic wavefronts in optical systems by an augmented stepalong method for vergences," Optom. Vis. Sci. 82, 923-32 (2005).

7. E. Acosta and R. Blendowske, "Paraxial optics of astigmatic systems: relations between the wavefront and the ray picture approaches," Optom. Vis. Sci. 84, E72 (2007).

8. H. Diepes and R. Blendowske, Optik und Technik der Brille (Optische Fachveröffentlichung GmbH, 2002), pp. 477-486.

9. L. Thibos, "Propagation of astigmatic wavefronts using power vectors," S. Afr. Optom. 62, 111-113 (2003).

10. K. P. Thompson, "Multinodal fifth-order optical aberrations of optical systems without rotational symmetry; the comatic aberrations,” J. Opt. Soc. Am. A 27, 1490-1504 (2010).

11. K. P. Thompson, "Real-ray-based method for locating individual surface aberration field centers in imaging optical systems without rotational symmetry," J. Opt. Soc. Am. A 26, 1503-1517 (2009).

12. J. Arasa and J. Alda, "Real ray tracing," in Encyclopedia of Optical Engineering (Marcel Dekker, 2004), doi:101081EEOE120027488.

13. J. Alda and J. Arasa, "Paraxial ray tracing," in Encyclopedia of Optical Engineering (Marcel Dekker, 2004), doi:101081EEOE120009643.

14. G. Dai, Wavefront Optics for Vision Correction (SPIE, 2008), pp. 129-255.

15. K. Dillon, "Bilinear wavefront transformation," J. Opt. Soc. Am. A 26, 1839-1846 (2009)

16. J. Schwiegerling, "Scaling Zernike expansion coefficients to different pupil sizes," J. Opt. Soc. Am. A 19, 1937-1945 (2002).

17. C. Campbell, "Matrix method to find a new set of Zernike coefficients from an original set when the aperture radius is changed," J. Opt. Soc. Am. A 20, 209-217 (2003).

18. G. Dai, "Scaling Zernike expansions coefficients to smaller pupil sizes: a simpler formula," J. Opt. Soc. Am. 23, 539-543 (2006).

19. H. Shu, L. Luo, and G. Han, "General method to derive the relationship between two sets of Zernike coefficients corresponding to different aperture sizes," J. Opt. Soc. Am. 23, 1960-1968 (2006).

20. S. Bara, J. Arines, J. Ares, and P. Prado, "Direct transformation of Zernike eye aberration coefficients between scaled, rotated and/or displace pupils,” J. Opt. Soc. Am. 23, 2061-2066 (2006).

21. L. Lundström and P. Unsbo, "Transformation of Zernike coefficients: scaled, translated and rotated wavefronts with circular and elliptical pupils,” J. Opt. Soc. Am. 24, 569-577 (2007). 
22. A. Guirao, D. Williams, and I. Cox, "Effect of the rotation and translation on the expected benefit of an ideal method to correct the eye's high-order aberrations," J. Opt. Soc. Am. A 18, 1003-1015 (2001).

23. G. Dai, C. Campbell, L. Chen, H. Zhao, and D. Chernyak, "Wavefront propagation from one plane to another with the use of Zernike polynomials and Taylor monomials," Appl. Opt. 48, 477-488 (2009)

24. W. Harris, "Power vectors versus power matrices, and the mathematical nature of dioptric power," Optom. Vis. Sci. 84, 1060-1063 (2007).
25. W. Harris, "Dioptric power: its nature and its representation in three- and four-dimensional space," Optom. Vis. Sci. 74 349-366 (1997).

26. L. Thibos, W. Wheeler, and D. Horner, "Power vectors: an application of fourier analysis to the description and statistical analysis of refractive error," Optom. Vis. Sci. 74, 367-375 (1997)

27. G. M. Dai, "Wavefront expansion basis functions and their relationships," J. Opt. Soc. Am. A 23, 1657-1666 (2006).

28. G.-M. Dai, "Wavefront expansion basis functions and their relationships: errata," J. Opt. Soc. Am. A 23, 2970-2971 (2006). 